Title Page

\title{
Utility of self-destructing CRISPR/Cas constructs for targeted gene editing in the retina
}

Fan $\mathrm{Li}^{1,2,3}$, Sandy S.C. Hung ${ }^{2}$, Mohd Khairul Nizam Mohd Khalid ${ }^{1}$, Jiang-Hui Wang ${ }^{2,4}$, Vicki Chrysostomou ${ }^{2,4}$, Vickie H.Y. Wong ${ }^{5}$, Vikrant Singh ${ }^{1}$, Kristof Wing ${ }^{1}$, Leilei Tu ${ }^{6}$, James A. Bender $^{7}$, Alice Pébay ${ }^{2,4,8}$, Anna E. King ${ }^{7}$, Anthony L. Cook ${ }^{7}$, Raymond C.B. Wong ${ }^{2,4}$, Bang V. Bui $^{5}$, Alex W. Hewitt ${ }^{1,2,4,9}$, Guei-Sheung Liu ${ }^{1,2,4,6,9}$

${ }^{1}$ Menzies Institute for Medical Research, University of Tasmania, Hobart, Tasmania, Australia

${ }^{2}$ Centre for Eye Research Australia, Royal Victorian Eye and Ear Hospital, East Melbourne, Victoria, Australia

${ }^{3}$ State Key Laboratory of Ophthalmology, Zhongshan Ophthalmic Centre, Sun Yat-sen University, Guangzhou, Guangdong, China

${ }^{4}$ Ophthalmology, Department of Surgery, University of Melbourne, East Melbourne, Victoria, Australia

${ }^{5}$ Department of Optometry and Vision Sciences, University of Melbourne, Parkville, Victoria, Australia

${ }^{6}$ Department of Ophthalmology, Jinan University, Guangzhou, Guangdong, China

${ }^{7}$ Wicking Dementia Research and Education Centre, University of Tasmania, Hobart, Tasmania, Australia

${ }^{8}$ Department of Anatomy and Neuroscience, University of Melbourne, Parkville, Victoria, Australia

${ }^{9}$ These authors contributed equally as senior authors.

Correspondence and requests for materials should be addressed to GSL (rickliu0817@gmail.com) and AWH (hewitt.alex@gmail.com) 


\section{Abstract}

Safe delivery of CRISPR/Cas endonucleases remains one of the major barriers to the widespread application of in vivo genome editing. We previously reported the utility of adeno-associated virus (AAV)-mediated CRISPR/Cas genome editing in the retina; however, with this type of viral delivery system, active endonucleases will remain in the retina for an extended period, making genotoxicity a significant consideration in clinical applications. To address this issue, we have designed a self-destructing "kamikaze" CRISPR/Cas system that disrupts the Cas enzyme itself following expression. Four guide RNAs (sgRNAs) were initially designed to target Streptococcus pyogenes Cas9 (SpCas9) and after in situ validation, the selected sgRNAs were cloned into a dual AAV vector. One construct was used to deliver SpCas9 and the other delivered sgRNAs directed against SpCas9 and the target locus (yellow fluorescent protein, YFP), in the presence of mCherry. Both constructs were packaged into AAV2 vectors and intravitreally administered in C57BL/6 and Thy1-YFP transgenic mice. After 8 weeks the expression of SpCas9 and the efficacy of YFP gene disruption was quantified. A reduction of SpCas9 mRNA was found in retinas treated with AAV2-mediated-YFP/SpCas9 targeting CRISPR/Cas compared to those treated with YFP targeting CRISPR/Cas alone. We also show that AAV2-mediated delivery of YFP/SpCas9 targeting CRISPR/Cas significantly reduced the number of YFP fluorescent cells among mCherry-expressing cells ( 85.5\% reduction compared to LacZ/SpCas9 targeting CRISPR/Cas) in the transfected retina of Thy1-YFP transgenic mice. In conclusion, our data suggest that a self-destructive "kamikaze" CRISPR/Cas system can be used as a robust tool for genome editing in the retina, without compromising on-target efficiency. 


\section{Introduction}

Inherited retinal diseases are disabling disorders of visual function that affect millions of people worldwide. With the development of next-generation sequencing and better molecular diagnostic techniques, numerous genetic variants across many loci have been definitively associated with inherited retinal diseases ${ }^{1,2}$. Despite this increase in our understanding of genetic aetiology and potential therapeutic targets, there remains no effective treatment for the majority of inherited retinal diseases. Although significant progress in gene therapy has been achieved over the last two decades, there are few sustained, safe and effective ocular gene therapy for hereditary retinal diseases ${ }^{3-5}$.

Advances in genome editing techniques, in particular the recent advances in CRISPR/Cas technology ${ }^{6}$, has renewed excitement in ocular gene-based therapy. The CRISPR/Cas system has evolved in archaea and bacteria as a defence against viral intrusion and has been adapted to allow efficient editing of mammalian nuclear genomes ${ }^{6}$. CRISPR/Cas-based technology has proven to be a robust means for in vitro correction of genetic mutations in mammalian cells and is particularly attractive for treating inherited retinal diseases ${ }^{7}$. A number of in vivo studies in various animal models have yielded promising results for pre-emptive therapy for well-characterised monogenic ocular diseases. Bakondi et $a l^{8}$ and Latella et al. ${ }^{9}$ report that successful ablation of the mutated rhodopsin gene prevented retinal degeneration in rodent models of severe autosomal dominant retinitis pigmentosa following electroporation of the CRISPR/Cas9 system into the retina. More recently, Yu et al. ${ }^{10}$ demonstrated that CRISPR/Cas9-mediated disruption of a neural retina-specific leucine zipper protein (NRL) significantly improved rod survival and preserved cone function in a murine model of retinal degeneration. We were able to achieve high efficiency genome editing in mouse retina using a dual AAV2-mediated CRISPR/Cas9 system ${ }^{11}$. However, potentially deleterious effects of prolonged overexpression of CRISPR/Cas endonuclease including elevated off-target cleavage $\mathrm{e}^{12,13}$, and cellular immune responses ${ }^{14}$ remain important safety hurdles to clinical application.

To address this, we have designed a self-destructive "kamikaze" CRISPR/Cas system that disrupts the CRISPR/Cas gene after active protein expression (Figure 1). To determine the efficacy of in vivo genome editing by our "kamikaze" CRISPR/Cas construct, a SpCas9 targeting sgRNA module, together with a yellow fluorescent protein (YFP) targeting sgRNA, 
were packaged into a dual AAV2 vector system for intravitreal delivery in Thy1-YFP transgenic mice.

\section{Materials and Methods}

\section{Animals and housing}

All procedures were conducted according to the Association for Research in Vision and Ophthalmology Statement for the Use of Animals in Ophthalmic and Vision Research and the requirements of National Health and Medical Research Council of Australia (Australian Code of Practice for the Care and Use of Animals for Scientific Purposes). Ethics approval was obtained from the Animal Ethics Committees of the University of Tasmania (A14827) and St. Vincent's Hospital Melbourne (AEC 014/15). Thy1-YFP transgenic mice [B6.Cg$\mathrm{Tg}$ (Thy1-YFP)16Jrs/J] were obtained from the Jackson Laboratory (mouse stock number: 003709; Bar Harbor, ME, USA) and bred at the animal facility of the Menzies Institute for Medical Research (Hobart, TAS, Australia). C57BL/6 mice were purchased from the Animal Resources Centre (Perth, WA, Australia). Mice were housed under standard conditions $\left(20^{\circ} \mathrm{C}, 12 / 12\right.$-hour light/dark cycle) with ad libitum access to food and water.

\section{sgRNA design and vector construction}

Four sgRNAs targeting the SpCas9 sequence were designed using a web-based CRISPR design tool (http://crispr.mit.edu). CRISPR/Cas in situ testing was carried out by incubating the individual synthetic SpCas9 sgRNA or LacZ sgRNA alone with the recombinant SpCas9 protein (catalog no. M03865; New England Biolabs, Ipswich, MA, USA) and the pX551 plasmid (SpCas9 construct; kindly provided by Feng Zhang, Addgene \#60957). Samples were run on a $0.8 \%$ TAE agarose gel to visualize their cleavage efficiency for SpCas9. Agel (catalog no. R0552S; New England Biolabs) digested pX551 plasmid was used as a positive control. Four SpCas9 sgRNAs were then cloned into a pX552-CMV-GFP plasmid (modified from Addgene \#60958, by replacing the hSYN1 promoter with a CMV promoter) at the Sapl restriction site for in vitro validation. Subsequently, the selected SpCas9 sgRNA (SpCas9 sgRNA4) was sub-cloned into an AAV package plasmid ( $p$ X552-hSYN1-mCherry-YFP sgRNA2, sgRNA6 or pX552-LacZ sgRNA) at the Mlul (catalog no. R3198; New England Biolabs) restriction site to generate YFP or LacZ targeting kamikaze CRISPR/Cas9 construct. For in vitro validation, pX551-CMV-SpCas9 plasmid was modified from pX551 plasmid by replacing the MeCP2 promoter with a CMV promoter. 


\section{Cell culture and transfection}

Stable YFP expressing HEK293A cells were generated using a lentivirus as previously described ${ }^{11,15}$. HEK293A-YFP cells were maintained in Dulbecco's modified Eagle's media (DMEM; catalog no. 11965118; Life Technologies Australia, Scoresby, VIC, Australia) supplemented with $10 \%$ fetal calf serum (Sigma-Aldrich, St. Louis, MO, USA), 2 mM glutamine (catalog no. 2503008; Life Technologies Australia), $50 \mathrm{U} / \mathrm{mL}$ penicillinstreptomycin (catalog no. 15070063; Life Technologies Australia) in a humidified $5 \% \mathrm{CO}_{2}$ atmosphere at $37{ }^{\circ} \mathrm{C}$. Transfection was undertaken with FuGENE-HD transfection reagent (catalog no. E2311; Promega Australia, Alexandria, NSW, Australia) according to the manufacturer's instruction. Briefly, HEK293A-YFP cells were seeded onto a 6-well plate $\left(2.5 \times 10^{5}\right.$ per well) 24 hours before transfection. A mixture of $7.5 \mu \mathrm{L}$ FuGENE-HD transfection reagent with $1500 \mathrm{ng}$ plasmid $(750 \mathrm{ng} /$ plasmid was used in dual plasmid transfection) in $150 \mu \mathrm{L}$ Opti-MEM (catalog no. 11058021; Life Technologies Australia) was added into each well. For in vitro validation of SpCas9 sgRNA, cells were collected for western blot analysis at day 3 after transfection; for in vitro time course analysis, cells were harvested at day 1, 2, 3, 5 and 7 days after transfection.

\section{Western blot analysis}

Cells were collected and lysed in ice-cold Cell Lysis Buffer (Catalog no.89900; Thermo Fisher Scientific, Waltham, MA, USA) and sonicated for 10 seconds by an ultrasonic cell disruptor (MISONIX Microson XL 2000; Qsonica, Newtown, CT, USA). Total protein was quantified by a Bio-Rad protein assay (Catalog no. 5000006; BIO-RAD, Hercules, CA, USA) using a microplate reader (Infinite M1000 Pro; TECAN, Männedorf, Switzerland). A total of $10 \mu \mathrm{g}$ protein samples were separated using NuPAGE ${ }^{\mathrm{TM}}$ Novex $^{\mathrm{TM}} 4-12 \%$ Bis-Tris Protein Gels (catalog no. NP0321BOX; Life Technologies Australia) and transferred to polyvinylidene fluoride membranes (catalog no. 162-0177; BIO-RAD) using the XCell II ${ }^{\text {TM }}$ Blot Module (Life Technologies Australia). Membranes were blocked with 5\% skim milk in TBS-T (10 mM Tris, $150 \mathrm{mM} \mathrm{NaCl}$, and $0.05 \%$ Tween-20) at room temperature for 1 hour and then incubated with mouse monoclonal SpCas9 antibody (1:1000 dilution; MAC133, lot number 2591899; Millipore, Billerica, MA, USA) or mouse monoclonal $\beta$-actin antibody (1:2000 dilution; MAB 1501, lot number 2722855; Millipore) at room temperature for 1 hour. Membranes were washed, further incubated with horseradish peroxidase-conjugated goat anti-mouse 
secondary antibody (1:5000 dilution; catalog no. A-11045; Life Technologies Australia) at room temperature for 1 hour, and developed using the Amersham ECL Prime Western Blotting Detection kit (catalog no. RPN2232; GE Healthcare Australia, Parramatta, NSW, Australia). The relative levels of SpCas9 protein of each sample was quantified using densitometry analysis (ImageJ software-gels analysis) with normalization to $\beta$-actin.

\section{YFP detection}

YFP expressing HEK293A cells were trypsinized and harvested in PBS. Cells were stained with DAPI $(5 \mu \mathrm{g} / \mathrm{mL})$ to exclude the dead cells. The percentage of YFP positive cells was then analyzed from live cell population with a Flow Cytometer (BD FACS Canto II; BD bioscience, Sparks, MD, USA) and data were analyzed using FACS analysis software (FlowJo ${ }^{\oplus}$; FlowJo LLC, Ashland, OR, USA).

\section{AAV production}

Recombinant AAV2 viruses were produced in HEK293D cells (kindly provided by lan Alexander, Children's Medical Research Institute, Westmead, NSW, Australia) packaging either pX551 plasmid, containing SpCas9, or pX552-mCherry plasmid with the respective sgRNAs (SpCas9, YFP or LacZ sgRNA), pseudoserotyped with the AAV2 capsid (pXX2) ${ }^{16}$, and purified using a AAV2pro Purification Kit (catalog no. 6232; Clontech Laboratories, Mountain View, CA, USA) as previously described ${ }^{11,15}$. Viral titer was determined by realtime quantitative PCR using a Fast SYBR Green Master Mix (catalog no. 4385612; Life Technologies Australia) with the pX551 or pX552 forward and reverse primers (Supplementary Table 1).

\section{Off-target effects assessment}

Off-target mutagenesis for the YFP targeting sgRNAs, YFP sgRNA2 and YFP sgRNA6, as well as SpCas9 targeting sgRNA, SpCas9 sgRNA4, were assessed using online prediction tool (Cas-OFFinder) in the Mus musculus genome. Potential off-target effects were further investigated using the method described by Chen et al. ${ }^{17}$ Briefly, 10 guide RNA sequences were designed to contain a single-nucleotide mismatch between the spacer and protospacer target at the YFP gene locus targeted by YFP sgRNA6, spanning from $5^{\prime}$ to the protospacer adjacent motif. These mismatch-containing guide RNAs (sgRNA M2 M20) were generated in conventional and kamikaze AAV-CRISPR/Cas9 systems and transfected in the YFP expressing HEK293A cells. Genomic DNA was extracted (catalog no. D4068; 
Zymo Research, Irvine, CA, USA) at day 10 post transfection and T7E1 assay was performed at YFP targeting loci with forward and reverse primers (supplementary table 1) by using an EnGen Mutation Detection Kit (catalog no. E3321; New England Biolabs). Mismatch cleavages by T7E1 were analyzed by Image J. For Sanger sequencing, PCR amplicon (885 bp) from genomic DNA was purified using DNA Clean \& Concentrator kit (catalog no. D4033; Zymo Research) and were sequenced in-house on the Applied Biosystems 3500 Genetic Analyzer (Thermo Fisher Scientific) using YFP reverse sequencing primer (Supplementary table 1). Sanger files were analyzed for insertions and deletions using the inference of CRISPR edits (ICE) tool (https://ice.synthego.com/\#/).

Intravitreal Injection

For our in vivo time course analysis, a total of 76 C57BL/ 6 adult mice, aged between 12 and 14 weeks were randomly separated into two groups, to receive either AAV2SpCas9+AAV2-YFP sgRNA2 $(n=39)$ or AAV2-SpCas9+AAV2-SpCas9 sgRNA/YFP sgRNA2 ( $n=$ 37). For the YFP disruption experiments, a total of 49 Thy1-YFP transgenic mice, aged between 16 and 20 weeks, were randomly allocated into three groups; those receiving AAV2-SpCas9+AAV2-YFP sgRNA2 ( $n=17$ ), AAV2-SpCas9+AAV2-SpCas9 sgRNA/YFP sgRNA2 $(n=17)$ or AAV2-SpCas9+AAV2-SpCas9 sgRNA/LacZ sgRNA ( $n=15)$. In addition, another 29 Thy1-YFP transgenic mice were used to test different YFP sgRNAs. These mice were randomly allocated into three groups; those receiving AAV2-SpCas9+AAV2-YFP sgRNA6 ( $n=$ 9), AAV2-SpCas9+AAV2-SpCas9 sgRNA/YFP sgRNA6 $(n=10)$ or AAV2-SpCas9+AAV2-SpCas9 sgRNA/LacZ sgRNA $(n=10)$.

Mice were anesthetized by intraperitoneal injection of ketamine $(60 \mathrm{mg} / \mathrm{kg})$ and xylazine $(10 \mathrm{mg} / \mathrm{kg})^{11}$. Intravitreal injection was performed under a surgical microscope. After a small puncture was made through the conjunctiva and sclera using a 30-gauge needle, a hand-pulled glass micropipette connected to a $10 \mu \mathrm{L}$ Hamilton syringe (BioStrategy, Broadmeadows, VIC, Australia) was inserted into the vitreous. A total of $1 \mu \mathrm{L}$ dual-viral suspension (AAV2-SpCas9: $2.5 \times 10^{9}$ vector genomes $(\mathrm{vg}) / \mu \mathrm{L}$ with AAV2-YFP sgRNA: $2.5 \times 10^{9} \mathrm{vg} / \mu \mathrm{L}$, AAV2-SpCas9 sgRNA/YFP-sgRNA: $2.5 \times 10^{9} \mathrm{vg} / \mu \mathrm{L}$ or AAV2-SpCas9 sgRNA/LacZ sgRNA: $2.5 \times 10^{9} \mathrm{vg} / \mu \mathrm{L}$ ) was injected into one eye of each mouse using a UMP32 Ultra Micro Pump (World Precision Instruments, Sarasota, FL, USA) at a rate of $200 \mathrm{~nL} / \mathrm{s}$. Any issues with the injection, including backflow upon removal of the needle, 
haemorrhaging of the external or internal vessels, retinal detachment was recorded, and eyes were excluded from the study.

\section{Electroretinography (ERG) and Optical Coherence Tomography (OCT)}

At 8 weeks following injection, mice underwent overnight dark-adaptation ( 12 hours), followed by electroretinography assessment under fully dark-adapted conditions. Details for functional assessment have been outlined previously ${ }^{11,18}$, with the exception that the reference chloride silver electrode was placed around the outside of the eye. ERG analysis was as previously described ${ }^{11,18}$ and returned the photoreceptor (a-wave), bipolar cell (bwave), and ganglion cell dominated (scotopic threshold response, STR) components of the waveform. Group data are given as mean ( \pm standard error of the mean [SEM]).

Following ERG recordings, retinal images were obtained using a spectral domainOCT (Bioptigen, Inc., Morrisville, NC, USA). Mice were positioned to capture Optic Nerve Head (ONH) centred $1.4 \mathrm{~mm}$-wide horizontal B-scans (consisting of $1000 \mathrm{~A}$-scans). ImageJ software (https://imagej.nih.gov/ij/) was used in a masked fashion to quantify total retinal thickness (from the inner limiting to Bruch's membrane), retinal nerve fibre layer thickness (from the inner limiting membrane to the inner aspect of the inner plexiform layer) and outer retinal thickness (from Bruch's membrane to the outer plexiform layer) in each eye.

\section{Retinal flat-mount, imaging and counting}

Eyes were removed, fixed in ice-cold $4 \%$ paraformaldehyde for 1 hour and dissected under a dissecting microscope. After removing the cornea, iris and lens, four equally spaced radial relaxing incisions, extending two thirds of the way from the retinal periphery to the $\mathrm{ONH}$, were made. The sclera and choroid were then removed along with residual vitreous and hyaloid vessels, leaving only the retina. The fully dissected retina was stained with NucBlue $^{\mathrm{TM}}$ Live ReadyProbes ${ }^{\mathrm{TM}}$ Reagent (catalog no. R37605; Life Technologies Australia) as a nuclear counterstain. Retinal images were captured by a fluorescence microscope (Zeiss Axio Imager Microscope; Carl-Zeiss-Strasse, Oberkochen, Germany) equipped with a charge-coupled digital camera (Axiocam MRm, Zeiss) and image acquisition software (ZEN2, Zeiss) as previously described ${ }^{11}$.

The efficiency of YFP disruption was quantified using individual fluorescent images captured at $\times 400$ magnification. A total of 24 images from three flat-mounted eyes treated with SpCas9 sgRNA/LacZ sgRNA, 36 images from five flat-mounted eyes treated with 
SpCas9 sgRNA/YFP sgRNA2 and 36 images from five flat-mounted eyes treated with YFP sgRNA2 were quantified manually using ImageJ v1.49 by an experienced grader (FL), masked to treatment status. For the second experiment with YFP sgRNA6, 16 images from three flat-mounted eyes treated with SpCas9 sgRNA/LacZ sgRNA, 38 images from five flatmounted eyes treated with SpCas9 sgRNA/YFP sgRNA6 and 36 images from 6 flat-mounted eyes treated with YFP sgRNA6 were quantified. Efficiency for each treatment group was determined as the proportion of YFP-negative cells relative to mCherry-expressing cells as previously described ${ }^{11}$.

\section{Statistical Analysis}

All statistical analyses were performed using Prism 7 software (GraphPad Software, Inc., La Jolla, CA, USA). Group data are represented as mean \pm SEM unless otherwise noted. Mean data were analyzed with unpaired t-tests, one-way or two-way analysis of variance (ANOVA) followed by post-hoc analysis (GraphPad Prism 7.0). A value of $p<0.05$ was taken to be statistically significant.

\section{Results}

\section{Generation and validation of kamikaze CRISPR/Cas construct in vitro.}

We first validated four sgRNAs (Figure 2A) for SpCas9 targeting using an in situ cleavage assay. Robust cleavage of the SpCas9 plasmid (pX551) was found when each of the four designed SpCas9 sgRNAs were introduced to recombinant SpCas9 protein (Figure 2B). We further confirmed the efficacy of SpCas 9 gene perturbations by transfection of the SpCas9 expression construct ( $p$ X551-CMV-SpCas9) together with SpCas9 targeting CRISPR/Cas constructs carrying different SpCas9 sgRNA (pX552-SpCas9 sgRNA1-4) into HEK293A cells. SpCas9 sgRNA4 had a clear destructive effect on SpCas9 (Figure 2C), reduction of SpCas9 protein, as well as having a lower off-target score against the human genome as predicted by a web-based CRISPR design program (http://crispr.mit.edu). A time course analysis showed that SpCas9 protein was progressively reduced in cells following the transfection of selected SpCas9 targeting CRISPR/Cas construct (pX552-SpCas9 sgRNA4) compared to LacZ sgRNA control ( $p X 552$-LacZ sgRNA; $p<0.05$; Figures 2D and 2E).

We next re-engineered our kamikaze CRISPR/Cas construct with YFP targeting sgRNAs or a LacZ targeting sgRNA (Figure 3A), and the efficacy of YFP gene disruption in the YFP-expressing HEK293A cells was assessed. Two days after transfection, we observed 
a reduction of SpCas9 protein in cells that had received the kamikaze CRISPR/Cas construct compared to those cells that had received the conventional CRISPR/Cas construct (Figure 3B). In terms of efficiency, the percentage of YFP-expressing cells was significantly reduced in cells transfected with the YFP targeting kamikaze CRISPR/Cas constructs (SpCas9 sgRNA/YFP sgRNA2: $10.0 \pm 1.0 \%$ and SpCas9 sgRNA/YFP sgRNA6: $5.6 \pm 0.4 \%$ respectively), compared to LacZ targeting kamikaze (SpCas9 sgRNA/LacZ sgRNA: $85.9 \pm 1.3 \%$ ) or LacZ targeting (LacZ sgRNA: $86.0 \pm 1.4 \%$ ) CRISPR/Cas construct at 10 days after transfection (Figures 3C and 3D). Similarly, a lower percentage of YFP expressed cells was also found in cells transfected with the YFP targeting CRISPR/Cas construct (YFP sgRNA2: $16.7 \pm 2.7 \%$ and YFP sgRNA6: $7.4 \pm 1.4 \%$ respectively; Figures 3 C and $3 D$ ).

In silico prediction of off-target sites for YFP sgRNA6 and SpCas9 sgRNA4 did not produce any significant candidates for testing, as both YFP and SpCas9 sequences were not found endogenously in the mouse genome. Next, we created a series of putative off-target sites by introducing single-nucleotide mismatch along YFP sgRNA6 sequence and compared the editing activity of conventional and kamikaze CRISPR/Cas systems (Figure 4A). A similar frequency of indels was induced by conventional or kamikaze CRISPR/Cas systems for knockout of YFP (Supplementary Figure 1). Almost all guide RNAs with singlenucleotide mismatches displayed editing activity at day 10 (Figure 4B) with the highest editing produced by a mismatch located distal to the PAM (M18) at day 10. A statistically reduction of editing activity at M2-M8 and M20 mutant guide RNA positions was observed with our kamikaze CRISPR/Cas system compared to the conventional CRISPR/Cas system (Figure 4C).

In vivo delivery of kamikaze CRISPR/Cas construct in the mouse retina.

To evaluate whether the reduction of SpCas9 expression by the kamikaze CRISPR/Cas construct compromises on-target editing efficiency Thy1-YFP mice received a single intravitreal injection of a dual viral suspension of AAV2-SpCas9 along with the YFP targeting kamikaze CRISPR/Cas construct (AAV2-SpCas9 sgRNA/YFP sgRNA) or the LacZ targeting kamikaze CRISPR/Cas construct (AAV2-SpCas9 sgRNA/LacZ sgRNA) or a single YFP targeting CRISPR/Cas construct as a positive control (AAV2-YFP sgRNA2). The procedures for our in vivo study is shown in Figure 5A. Eight weeks following treatment, images from the retinal flat-mounts showed that there were fewer YFP-positive cells among mCherry 
positive cells in mice that had received either AAV2-SpCas9 sgRNA/YFP sgRNA or AAV2-YFP sgRNA2 compared to AAV2-SpCas9 sgRNA/LacZ sgRNA (Figure 5B). Specifically, the proportion of retinal $\mathrm{YFP} / \mathrm{mCherry}$-expressing cells was reduced to $5.5 \pm 1.4 \%$ in AAV2SpCas9 sgRNA/YFP sgRNA2-treated retina and $7.3 \pm 1.3 \%$ in AAV2-YFP sgRNA2-treated retina, compared with $38.2 \pm 1.7 \%$ in AAV2-SpCas9 sgRNA/LacZ sgRNA treated eyes. Overall, there was a $85.5 \%(95 \% \mathrm{Cl}: 78.4-92.6)$ and $80.9 \%(95 \% \mathrm{Cl}: 74.3-87.5)$ reduction in YFP positive cells in AAV2-SpCas9 sgRNA/YFP sgRNA- and AAV2-YFP sgRNA2-treated retinas, respectively, compared to AAV2-SpCas9 sgRNA/LacZ sgRNA-treated eyes (Figure 5C). No significant difference in the percentage of YFP disruption was found in between AAV2-YFP sgRNA2- and AAV2-SpCas9 sgRNA/YFP sgRNA2-treated retinas ( $P=0.62$; Figure $5 C$ ). This was confirmed by using an alternate YFP targeting sgRNA (YFP sgRNA6), where the proportion of retinal YFP/mCherry-expressing cells was $17.0 \pm 1.3 \%$ in AAV2-SpCas 9 sgRNA/YFP sgRNA6-treated retina and 20.6 $1.2 \%$ in AAV2-YFP sgRNA6-treated retina, compared with $40.8 \pm 2.0 \%$ AAV2-SpCas9 sgRNA/LacZ sgRNA-treated eyes. This represents a relative reduction of $49.5 \%$ (95\% Cl: $43.5-55.5)$ and $58.3 \%(95 \% \mathrm{Cl}: 56.4-62.0)$ in AAV2SpCas9 sgRNA/YFP sgRNA6- and AAV2-YFP sgRNA6-treated retinas compared to those that had received AAV2-SpCas9 sgRNA/LacZ sgRNA, respectively (Supplementary Figure 2).

Furthermore, SpCas9 gene perturbations in vivo were confirmed by qPCR. A time course analysis showed that SpCas9 mRNA was significantly reduced in the retinas following the treatment of AAV2-SpCas9 sgRNA/YFP sgRNA2 compared to AAV2-YFP sgRNA2 ( $<<0.05$ at week 8; Supplementary Figure 3).

Retinal function and structure assessment by electroretinography (ERG) and optical coherence tomography (OCT).

To evaluate the effect of our "kamikaze" CRISPR/Cas construct on retinal function and structure, ERG and OCT were performed at 8 weeks after intravitreal injection of viral suspensions in Thy1-YFP mouse. Group averaged waveforms elicited using bright and dim flashes of light along with the group averaged data from eye injected with YFP targeting kamikaze-CRISPR/Cas constructs (AAV2-SpCas9 sgRNA/YFP sgRNA2, Figures 6A and 6B) and YFP targeting CRISPR/Cas constructs (AAV2-YFP sgRNA2, Figures 6E and 6F) suggest that both treatments affected retinal function when compared with the contralateral control eyes (Figure 6A-F and Supplementary Figures 4-6). LacZ targeting kamikaze-CRISPR/Cas 
construct (AAV2-SpCas9 sgRNA/LacZ sgRNA) treated eyes retained normal retinal function (Figure 6C and 6D). OCT analysis suggest that none of the CRISPR/Cas constructs negatively impacted retinal structure, as there were no significant differences in retinal nerve fibre layer and total retinal thickness between the vehicle and viral-injected eyes of all three groups (Figure 6G-1).

\section{Discussion}

This study builds on our recent work using AAV2-mediated CRISPR/Cas to edit genes in mouse retina ${ }^{11}$. While CRISPR/Cas9-mediated genome editing has shown promise for correcting disease-causing mutations, the potential for genotoxic effects with prolonged expression of CRISPR/Cas9 poses a significant barrier to the clinical utility of this technology. Several strategies have been employed in an attempt to avoid off-target cleavage, including improved guide RNA design ${ }^{19,20}$, or modification of Cas9 enzymes ${ }^{21,22}$. Such approaches do not avoid accumulation of Cas9, which can increase the overall chance of off-target cleavage and immunological response. Our approach was to employ a selfdestructive CRISPR/Cas system that disrupts the CRISPR/Cas enzyme itself after the active protein has been expressed. Unlike other approaches, most of which act to control the activity of the CRISPR/Cas system via chemical ${ }^{23,24}$, and biophysical ${ }^{25,26}$ modulation of Cas9, our kamikaze CRISPR/Cas system can significantly reduce accumulation of Cas9 protein in vitro and disrupt AAV-Cas9 construct in vivo, without dramatically compromising the efficiency of on-target editing. This approach is similar to that used by Merienne and colleagues $^{27}$ who demonstrated that progressively inactivating the nuclease using a Cas 9 self-inactivating editing system resulted in a lower frequency of off-target cleavage in human iPSCs-derived neurons in vitro and in mouse brains via lentiviral-mediated in vivo delivery. Chen et al. has also been tested similar self-restrictive CRISPR/Cas system in vitro ${ }^{17}$. While these previous works have shown the feasibility of a self-destructive CRISPR/Cas system, our study highlights the effectiveness of an AAV-mediated selfdestructive CRISPR/Cas system for in vivo genome editing in the retina.

We observed similar efficiencies in YFP gene perturbation between conventional and kamikaze CRISPR/Cas system, but also found differences between in vitro and in vivo models, especially in SpCas9 sgRNA/YFP sgRNA6 construct (\% YFP reduction in vitro: 93.5\% vs in vivo: $49.5 \%$ ) and YFP sgRNA6 construct (\% YFP reduction in vitro: $91.4 \%$ vs in vivo: 
$58.3 \%)$. This difference may be due to a dual AAV2 vector system was employed to deliver the kamikaze CRISPR/Cas construct in vivo. We and others have recently demonstrated that CRISPR/Cas9 delivered using a dual AAV2 vector can effectively edit the genome in a number of organs in adult mice ${ }^{11,28-30}$. However, expression of the CRISPR/Cas9 machinery requires the receipt of both Cas9 and sgRNA expression cassettes from two separate viral vectors, which may significantly reduce editing efficiency. Although a single viral vector system employing Cas9 orthologs such as $\mathrm{SaCas}^{31}$ or $\mathrm{CjCas} 9^{32}$ may provide better in vivo editing efficiency, dual-vector systems may still be required for mutation correction as they enable delivery of donor templates and appropriate promoter elements.

An unexpected reduction in retinal function was observed 8 weeks after injection of AAV2-SpCas9 sgRNA/YFP sgRNA2 or AAV2-YFP sRNA2. Interestingly, retinal function was unaffected in mice treated with AAV2-SpCas9 sgRNA/LacZ sgRNA, therefore, deficits in retinal function is not related to the SpCas9 sgRNA construct per se, but may be related to either off-target effects of YFP targeting sgRNA or accumulation of non-functional fluorescent proteins resulting from CRISPR/Cas9 editing. To further explore this possibility, firstly we tested a different YFP sgRNA (sgRNA6 which targets another region of the YFP sequence) in vivo. However, a significant decrease in retinal function was still present in AAV2-SpCas9 sgRNA/YFP sgRNA6 and AAV2-YFP sgRNA6 treated mice (Supplementary Figures 7-9). We then searched the mouse genome for potential off-target sites for two YFP sgRNAs and SpCas9 sgRNA by in silico prediction (Cas-OFFinder) and performed whole exome sequencing on the treated mouse retinae. No significant candidate genes for the off-target sites were found by in silico prediction or by whole exome sequencing (Supplementary Results). Therefore, it may be more likely that the reduction of retinal function arises from accumulation of mutated fluorescent proteins. Although fluorescence proteins such as GFP and YFP have been widely used in neuroscience research ${ }^{33}$, accumulation of non-functional proteins resulting from on-target deletions (indel) may lead a deleterious effect on retinal protein homeostasis ${ }^{34}$. Moreover, a recent study also indicated that large on-target deletions could lead to potential genotoxicity ${ }^{29}$. Whether such mechanisms account for the functional deficits observed in our study requires further investigation. Although no retinal toxicity was observed by over-expression of Cas 9 enzyme or through delivery of our self-destructive CRISPR/Cas system, this study was 
conducted over a relatively short period of time (8 weeks). As such the long-term safety profile and whether the potentially deleterious effects caused by prolonged overexpression of truncated gene products in the retina, as potentially caused by our selfdestructive CRISPR/Cas system, requires further investigation.

In summary, we describe and characterise a self-destructive "kamikaze" CRISPR/Cas system for in vivo genome editing in the retina. This self-destructive kamikaze CRISPR/Cas system can effectively reduce the expression of SpCas9 in the mouse retina, without substantially sacrificing on-target editing efficiency. Therefore, our AAV2mediated self-destructive CRISPR/Cas may be a useful tool for genome editing in the retina, especially when combined with high fidelity forms of CRISPR/Cas.

\section{Acknowledgments}

This work was supported by funding from a Bayer Global Ophthalmology Award, the Ophthalmic Research Institute of Australia, an Australian National Health and Medical Research Council (NHMRC) grant (\#1123329), a NHMRC Practitioner Fellowship (AWH, \#1103329), a NHMRC Senior Research Fellowship (AP, \#1154389), and an Australian Research Council Future Fellowship (AP, FT140100047). CERA receives Operational Infrastructure Support from the Victorian Government.

Author contributions: Conceptualization, F.L., S.H., A.W.H. and G.S.L. Methodology, F.L., S.H., B.V.B., A.W.H. and G.S.L. Formal Analysis, F.L., S.H., B.V.B., A.W.H. and G.S.L. Investigation, F.L., S.H., M.KN.M.K., J.H.W., V.C., V.W., V.S., L.T., K.W., J.A.B., R.W., B.V.B., A.W.H. and G.S.L. Resources, A.E.K., A.L.C., A.W.H. and G.S.L. Writing- Original Draft, F.L., A.W.H. and G.S.L. Writing- Review \& Editing, S.H., M.KN.M.K., J.H.W., A.P., A.E.K., A.L.C., R.W. and B.V.B. Visualization, F.L., A.W.H. and G.S.L. Supervision, A.W.H. and G.S.L. Project Administration, F.L., A.W.H. and G.S.L. Funding Acquisition, A.W.H. and G.S.L.

\section{Author Disclosure Statement}

The authors declare no competing financial interests exist. 


\section{References}

1. Neveling K, Collin RW, Gilissen C et al. Next-generation genetic testing for retinitis pigmentosa. Hum Mutat 2012;33:963-972.

2. Ratnapriya R, Swaroop A. Genetic architecture of retinal and macular degenerative diseases: the promise and challenges of next-generation sequencing. Genome Med 2013;5:84.

3. Bainbridge JW, Mehat MS, Sundaram V et al. Long-term effect of gene therapy on Leber's congenital amaurosis. N Engl J Med 2015;372:1887-1897.

4. Jacobson SG, Cideciyan AV, Roman AJ et al. Improvement and decline in vision with gene therapy in childhood blindness. N Engl J Med 2015;372:1920-1926.

5. Lee JH, Wang JH, Chen J et al. Gene therapy for visual loss: Opportunities and concerns. Prog Retin Eye Res 2018.

6. Jinek $M$, Chylinski $K$, Fonfara I et al. A programmable dual-RNA-guided DNA endonuclease in adaptive bacterial immunity. Science 2012;337:816-821.

7. Hung SSC, McCaughey T, Swann O et al. Genome engineering in ophthalmology: Application of CRISPR/Cas to the treatment of eye disease. Prog Retin Eye Res 2016;53:120.

8. Bakondi B, Lv W, Lu B et al. In Vivo CRISPR/Cas9 Gene Editing Corrects Retinal Dystrophy in the S334ter-3 Rat Model of Autosomal Dominant Retinitis Pigmentosa. Mol Ther 2016;24:556-563.

9. Latella MC, Di Salvo MT, Cocchiarella F et al. In vivo Editing of the Human Mutant Rhodopsin Gene by Electroporation of Plasmid-based CRISPR/Cas9 in the Mouse Retina. Mol Ther Nucleic Acids 2016;5:e389.

10. $\mathrm{Yu} \mathrm{W,} \mathrm{Mookherjee} \mathrm{S,} \mathrm{Chaitankar} \mathrm{V} \mathrm{et} \mathrm{al.} \mathrm{Nrl} \mathrm{knockdown} \mathrm{by} \mathrm{AAV-delivered}$ CRISPR/Cas9 prevents retinal degeneration in mice. Nat Commun 2017;8:14716. 
11. Hung SS, Chrysostomou V, Li F et al. AAV-Mediated CRISPR/Cas Gene Editing of Retinal Cells In Vivo. Invest Ophthalmol Vis Sci 2016;57:3470-3476.

12. Hsu PD, Scott DA, Weinstein JA et al. DNA targeting specificity of RNA-guided Cas9 nucleases. Nat Biotechnol 2013;31:827-832.

13. Fu Y, Sander JD, Reyon D et al. Improving CRISPR-Cas nuclease specificity using truncated guide RNAs. Nat Biotechnol 2014;32:279-284.

14. Wang D, Mou H, Li S et al. Adenovirus-Mediated Somatic Genome Editing of Pten by CRISPR/Cas9 in Mouse Liver in Spite of Cas9-Specific Immune Responses. Hum Gene Ther 2015;26:432-442.

15. Hung SS, Li F, Wang JH et al. Methods for In Vivo CRISPR/Cas Editing of the Adult Murine Retina. Methods Mol Biol 2018;1715:113-133.

16. Xiao X, Li J, Samulski RJ. Production of high-titer recombinant adeno-associated virus vectors in the absence of helper adenovirus. J Virol 1998;72:2224-2232.

17. Chen $Y$, Liu X, Zhang $Y$ et al. A Self-restricted CRISPR System to Reduce Off-target Effects. Mol Ther 2016;24:1508-1510.

18. Kong $\mathrm{YX}$, Crowston JG, Vingrys AJ et al. Functional changes in the retina during and after acute intraocular pressure elevation in mice. Invest Ophthalmol Vis Sci 2009;50:57325740 .

19. Ran FA, Hsu PD, Lin CY et al. Double nicking by RNA-guided CRISPR Cas9 for enhanced genome editing specificity. Cell 2013;154:1380-1389.

20. Moreno-Mateos MA, Vejnar CE, Beaudoin JD et al. CRISPRscan: designing highly efficient sgRNAs for CRISPR-Cas9 targeting in vivo. Nat Methods 2015;12:982-988.

21. Kleinstiver BP, Pattanayak V, Prew MS et al. High-fidelity CRISPR-Cas9 nucleases with no detectable genome-wide off-target effects. Nature 2016;529:490-495.

22. Slaymaker IM, Gao L, Zetsche B et al. Rationally engineered Cas9 nucleases with improved specificity. Science 2016;351:84-88. 
Page 17 of 41

23. Davis KM, Pattanayak V, Thompson DB et al. Small molecule-triggered Cas9 protein with improved genome-editing specificity. Nat Chem Biol 2015;11:316-318.

24. Dow LE, Fisher J, O'Rourke KP et al. Inducible in vivo genome editing with CRISPRCas9. Nat Biotechnol 2015;33:390-394.

25. Hemphill J, Borchardt EK, Brown K et al. Optical Control of CRISPR/Cas9 Gene Editing. J Am Chem Soc 2015;137:5642-5645.

26. Nguyen DP, Miyaoka Y, Gilbert LA et al. Ligand-binding domains of nuclear receptors facilitate tight control of split CRISPR activity. Nat Commun 2016;7:12009.

27. Merienne $\mathbf{N}$, Vachey $G$, de Longprez L et al. The Self-Inactivating KamiCas9 System for the Editing of CNS Disease Genes. Cell Rep 2017;20:2980-2991.

28. Swiech $L$, Heidenreich $M$, Banerjee $A$ et al. In vivo interrogation of gene function in the mammalian brain using CRISPR-Cas9. Nat Biotechnol 2015;33:102-106.

29. Yang $Y$, Wang $L$, Bell $P$ et al. A dual AAV system enables the Cas9-mediated correction of a metabolic liver disease in newborn mice. Nat Biotechnol 2016;34:334-338.

30. Bengtsson NE, Hall JK, Odom GL et al. Muscle-specific CRISPR/Cas9 dystrophin gene editing ameliorates pathophysiology in a mouse model for Duchenne muscular dystrophy. Nat Commun 2017;8:14454.

31. Ran FA, Cong L, Yan WX et al. In vivo genome editing using Staphylococcus aureus Cas9. Nature 2015;520:186-191.

32. Kim E, Koo T, Park SW et al. In vivo genome editing with a small Cas9 orthologue derived from Campylobacter jejuni. Nat Commun 2017;8:14500.

33. Nour M, Quiambao AB, Al-Ubaidi MR et al. Absence of functional and structural abnormalities associated with expression of EGFP in the retina. Invest Ophthalmol Vis Sci 2004;45:15-22.

34. Tzekov R, Stein L, Kaushal S. Protein misfolding and retinal degeneration. Cold Spring Harb Perspect Biol 2011;3:a007492. 
Page 18 of 41

\section{FIGURE LEGENDS}

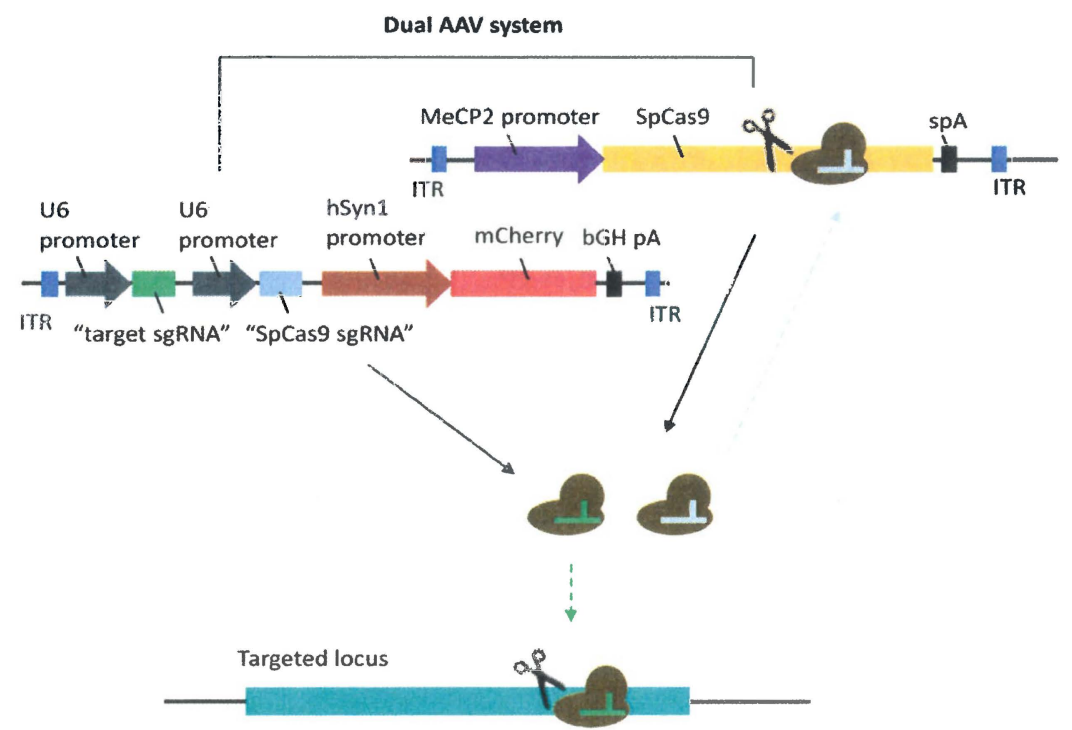

Figure 1. Schematics of Kamikaze CRISPR/Cas system. A dual AAV vector system was used. One viral vector was used to deliver SpCas9 and the other delivered sgRNAs against SpCas9 and the target locus (YFP), in the presence of mCherry. 
A SpCas9 target sequence

5 ....GTCGAAGCGTCCGACAAGAAGTACAGCATCGGCCTGGACATCGGCACCAACTCTGTGGGCTGGGCCGT...3. sgRnA1

sgRNA sequence PAM

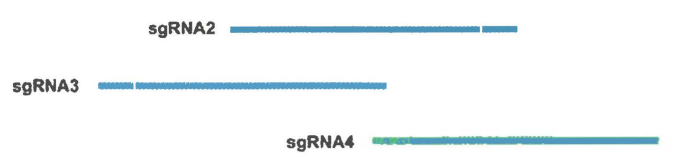

B

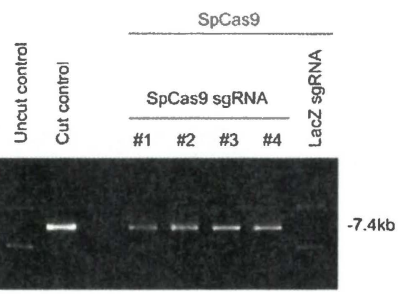

C

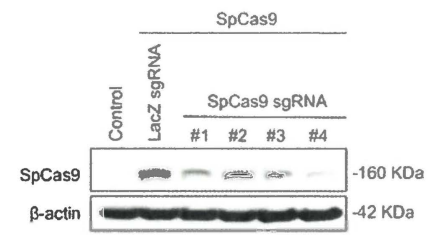

D
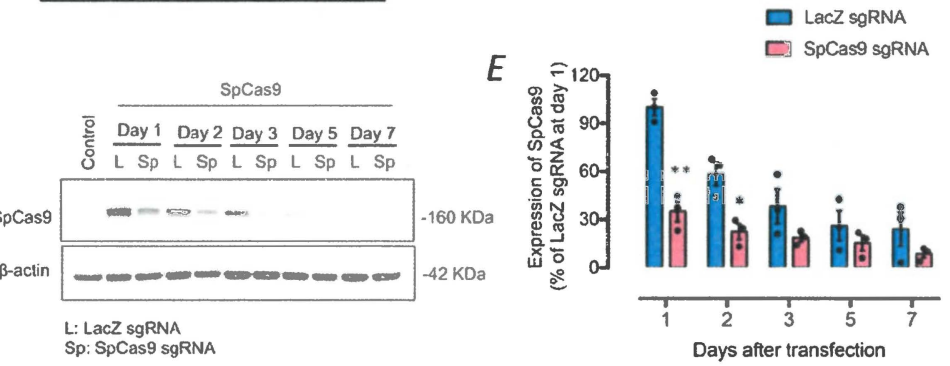

Figure 2. Design and validation of SpCas9 sgRNA. (A) Schematic diagram of SpCas9 sgRNA design. Blue: selected SpCas9 sgRNA targeted sites. Green: PAM sequences. (B) In situ validation of SpCas9 sgRNAs. (C) In vitro validation of SpCas9 sgRNAs. Representative western blot of SpCas9 protein expression in cells 2 days after co-transfected with SpCas9 and the individual SpCas9 sgRNA plasmids. (D) Representative western blot of the time course of SpCas9 expression. Cells were harvested on day 1, 2, 3, 5 and 7 after transfection with SpCas9 and selected SpCas9 sgRNA (\#4) plasmids. (E) Relative fold change of SpCas9 protein expression normalized to $\beta$-actin. Mean \pm SEM for 3 independent replicates. Statistical analysis between control and sgRNA-transfected group was performed using two-way ANOVA followed by Tukey's multiple comparisons test ( ${ }^{*} p<0.05,{ }^{*} p<0.001$ ). 
Page 20 of 41

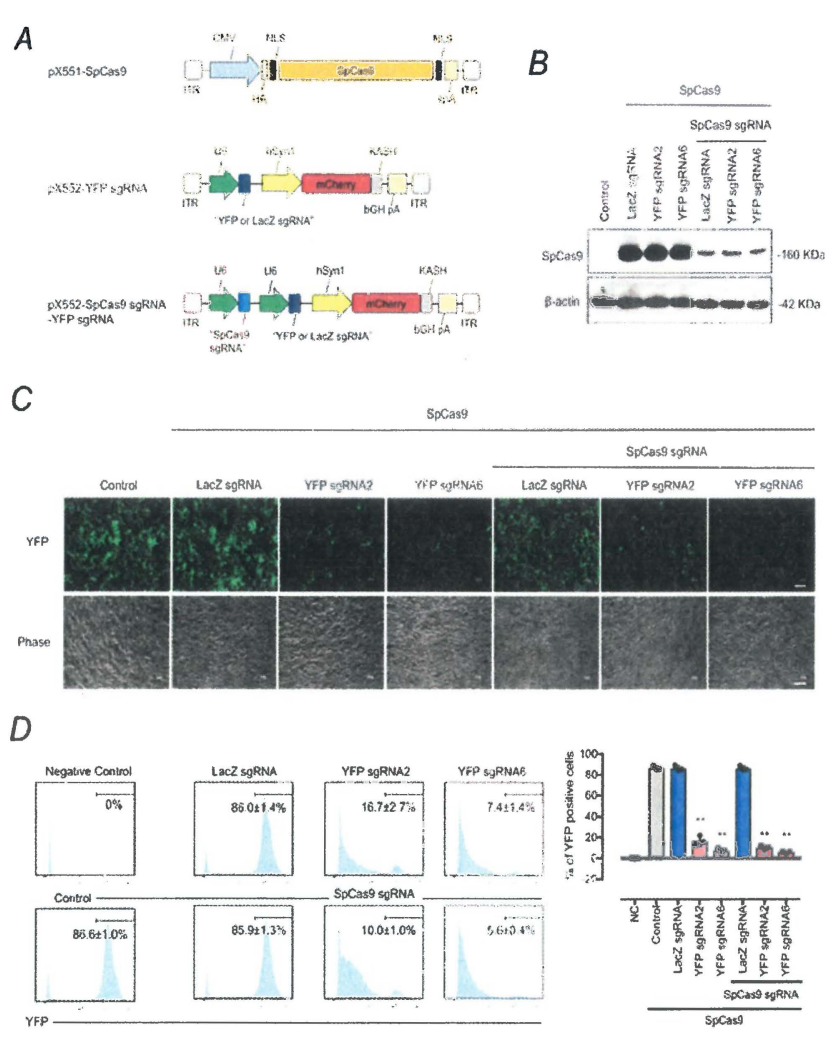

Figure 3. In vitro validation of kamikaze CRISPR/Cas construct. (A) Schematic of plasmid constructs for in vitro validation. (B) Representative Western blots of SpCas9 protein expression in cells co-transfected with SpCas9 and kamikaze (SpCas9 sgRNA/YFP sgRNA and SpCas9 sgRNA/LacZ sgRNA) or non-kamikaze (YFP sgRNA and LacZ sgRNA) constructs. (C) Representative images of YFP expression in cells co-transfected with kamikaze (SpCas9 sgRNA/YFP sgRNA and SpCas9 sgRNA/LacZ sgRNA) or non-kamikaze (YFP sgRNA and LacZ sgRNA) constructs. scale bar: $100 \mu \mathrm{m}$. (D) Percentage YFP disruption was assessed by FACS at 10 days after transfection. Mean \pm SEM for 3 independent replicates. Statistical analysis between control and sgRNA-transfected group was performed using one-way ANOVA followed by Tukey's multiple comparisons test $(* * p<0.001)$. 


\section{$B$}

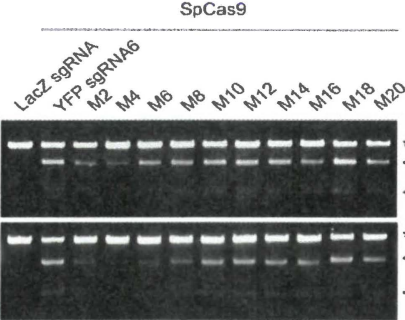

YFP sgRnAG

M4: CGTCGCGTCCAGCTCGAGC

M6: CGTCGCCGTCCAGCACGACC

M8: CGTCGCCGTCCACCTCGACC

M10: CGTCGCCGTCGAGCTCGACC

M12: CGICGCCGACCAGCTCGACC

M16:

M18: CGACCCCGTCCAGCTCGACC

M18: CGACGCCGTCCAGCTCGACC

C

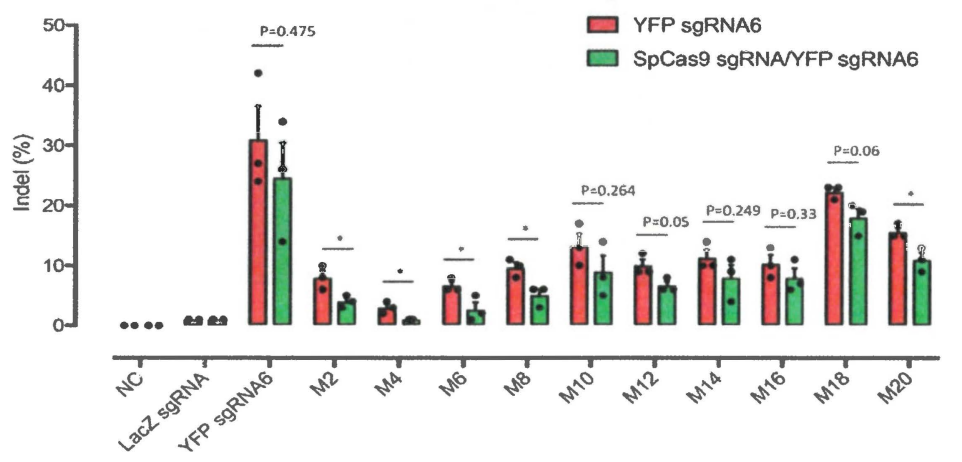

Figure 4. Kamikaze CRISPR/Cas reduced potential off-target in vitro. (A) Sequences of mismatch containing YFP-targeting guide RNAs. (B) T7E1 assay showing CRISPR/Cas9 editing activity of these single-nucleotide mismatches at day 10 after transfection. PCR products containing amplicons derived from mutant YFP or from wild-type YFP only are indicated by the arrows and asterisks, respectively. (C) Analysis of indels by ICE tool, indicating the difference in mismatch related CRISPR editing between kamikaze YFP targeting CRISPR/Cas system and conventional YFP targeting CRISRP/Cas system. Mean \pm SEM for 3 independent replicates. Statistical analysis between kamikaze YFP targeting CRISPR/Cas system and conventional YFP targeting CRISRP/Cas system was performed using two-tailed $t$-test $\left({ }^{*} \mathrm{p}<0.05\right)$. 


\section{$A$}

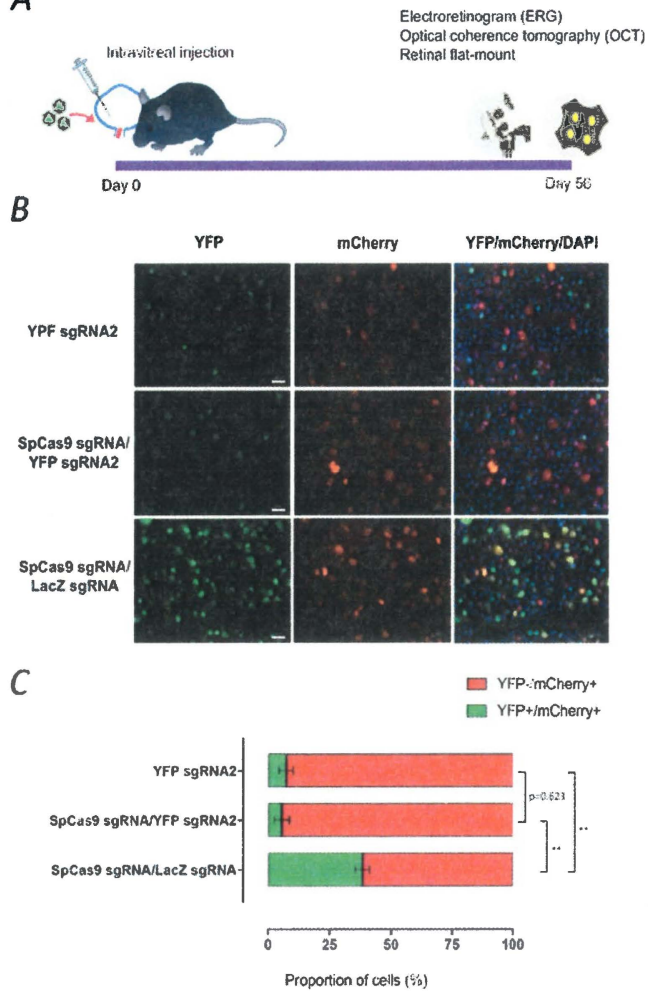

Figure 5. Kamikaze CRISPR/Cas-mediated genome editing of retinal cells in vivo. (A) High magnification of retinal flat-mount images, showing differences in YFP expression following AAV2-mediated delivery of SpCas9 sgRNA/YFP sgRNA ( $n=5)$, YFP sgRNA2 $(n=5)$ or SpCas9 sgRNA/LacZ sgRNA ( $n=3$ ). Scale bar: $20 \mu \mathrm{m}$. (B) Percentage YFP disruption was assessed by manual cell counting. Mean \pm SEM for 3-5 independent replicates. Statistical analysis between groups was performed using one-way ANOVA followed by Tukey's multiple comparisons

test $(* * p<0.001)$ 


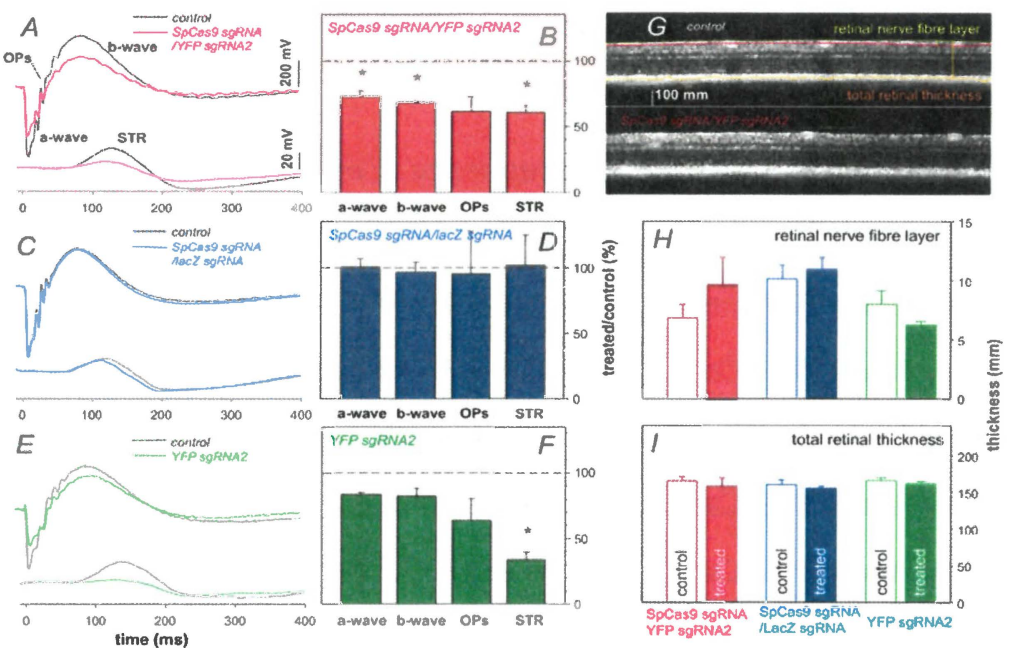

Figure 6. Effect of AAV2-mediated CRISPR/Cas administration on retinal function. Averaged ERG waveforms at selected intensities for control (black traces) and SpCas9 sgRNA/YFP sgRNA2 ( $n=4$, red traces; A), SpCas9 sgRNA/LacZ sgRNA $(n=4$, blue traces; C) and YFP sgRNA2 $(n=5$, green traces; E) injected eyes. Group average ( \pm SEM) photoreceptoral (a-wave), bipolar cell (b-wave), amacrine cell (oscillatory potentials, OPs) and ganglion cell (scotopic threshold response, STR) amplitude relative to contralateral control eyes (\%) for each group (B, D and F). Effect of SpCas9 sgRNA/YFP sgRNA2, SpCas9 sgRNA/LacZ sgRNA and YFP sgRNA2 on retinal structure measured with OCT (G). Group average $( \pm$ SEM) retinal nerve fibre layer thickness $(H)$ for SpCas9 sgRNA/YFP sgRNA2 treated (filled red, $n=4$ ) and their contralateral controls (unfilled red, $n=4$ ), SpCas9 sgRNA/LacZ sgRNA treated (filled blue, $n=4$ ) and their contralateral controls (unfilled blue, $n=4$ ) and YFP sgRNA2 treated (filled green, $n=5$ ) and their contralateral controls (unfilled green, $n=5$ ). Total retinal thickness (1). Statistical analysis between injected and control eyes was performed using two-tailed $t$-test $(* p<0.05)$. 
Supplementary Table 1. Sequence of primers for sgRNA cloning, vector construction, sequencing and $\mathrm{qPCR}$ analysis.

Supplementary Figure 1. Spectrum and frequency of indels for knockout of YFP .

Supplementary Figure 2. Quantification of YFP disruption in the retina.

Supplementary Figure 3. Time course of SpCas9 mRNA expression in the mouse retina.

Supplementary Figure 4. SpCas9 sgRNA/YFP sgRNA2 decreased retinal function.

Supplementary Figure 5. YFP sgRNA2 alone affects inner retinal function.

Supplementary Figure 6. SpCas9 sgRNA/LacZ sgRNA does not affect retinal function.

Supplementary Figure 7. SpCas9 sgRNA/YFP sgRNA6 decreased retinal function.

Supplementary Figure 8. YFP sgRNA6 alone affects inner retinal function.

Supplementary Figure 9. SpCas9 sgRNA/LacZ sgRNA does not affect retinal function. 
Supplementary methods:

- Quantitative PCR to detect SpCas9 expression in the retina.

- Genomic DNA extraction from mouse retinas and whole exome sequencing.

Supplementary Result: Whole exome sequencing showed no off-target effect of AAV2SpCas9 sgRNA/YFP sgRNA6.

Supplementary Information: Uncropped agarose gel and western blot images.

Supplementary methods:

Quantitative PCR to detect SpCas9 expression in the retina

Total RNA from mouse retinas were extracted and purified using commercial kits (RNeasy Mini Kit; catalog no. 74104; Qiagen, Chadstone, VIC, Australia) in accordance with the manufacturer's instructions. RNA was subsequently reverse-transcribed into complementary DNA (cDNA) using a high-capacity RT kit (catalog no. 4374996; Life Technologies Australia) and quantitative PCR was performed using a Fast SYBR Green Master Mix (catalog no. 4385612; Life Technologies Australia) with the SpCas9 forward and reverse primers as well as mCherry forward and reverse primers (Supplementary Table 1). The relative expression levels of SpCas9 was calculated using the ${ }^{\Delta \Delta} \mathrm{Ct}$ method with normalization to mCherry.

Genomic DNA extraction from mouse retinas and whole exome sequencing

Genomic DNA from two retinas treated with SpCas9 sgRNA/YFP sgRNA6 and two contralateral retinas were extracted using commercial Genomic DNA Purification Kits (QIAGEN, Puregene Core Kit A, Lot no. 8570335; RNase A Solution, Catalog no. 158922; Puregene Proteinase K, Catalog no. 158918) and dissolved in TE buffer. Whole exome sequencing and standard bioinformatics analyses were performed by Beijing Genomics Institute (BGI). Briefly, whole exome enrichment was carried out using the SureSelect ${ }^{\mathrm{XT}}$ Mouse All Exon Kit (Agilent) and sequencing was performed on NovaSeq 6000 with 150bp paired-end reads. Raw reads were filtered and the resulting high-quality, clean data were aligned to the mouse reference genome ( $\mathrm{mm10}$ ) using Burrows-Wheeler Aligner (BWA) v0.7.15 (https://www.ncbi.nlm.nih.gov/pubmed/20080505).

The variants were filtered using the following criteria: (1) SNVs were excluded to focus only on indels; (2) indel variants shared between treated retinas and contralateral untreated retinas were excluded; (3) known indels were excluded by comparing to dbSNP 
build 138; (4) homozygous indels were excluded. As for indel analysis, homology between sgRNA sequence and sequences around the indel site in the mouse reference genome were evaluated. Nucleotide sequences around the indel site were obtained using Mutalyzer

(https://mutalyzer.nl/) (https://onlinelibrary.wiley.com/doi/abs/10.1002/humu.20654). Alignment between the 20bp sgRNA sequence (both YFP sgRNA6 and SpCas9 sgRNA4 and in both sense and antisense orientation) (excluding the PAM) and the sequences provided by Mutalyzer were carried out using MAFFT (https://mafft.cbrc.jp/alignment/server/) (https://academic.oup.com/bib/advance-article/doi/10.1093/bib/bbx108/4106928). Offtarget effects were considered when MAFFT showed ungapped alignment between these sequences, with fewer than five nucleotide mismatches and presence of correct PAM site in the reference sequence.

Supplementary Result:

Whole exome sequencing showed no off-target effect of AAV2-SpCas9 sgRNA/YFP SgRNA6

Whole exome sequencing produced consistent coverage across all four samples (153.33155.68X). A total of 303 indel variants were detected in all four samples and 120 indels were shared between treated retinas and contralateral untreated retinas and were therefore removed. About 79 indels were unique in treated retinas and of these were removed due a match in dbSNP and/or were homozygous. When searching for putative off-target sequences around the remaining indels in the reference genome, we did not find any indels that could be associated with off-target effects of our kamikaze CRISPR/Cas system. 
Supplementary Table 1. Sequence of primers for sgRNA cloning, vector construction, sequencing and $\mathrm{qPCR}$ analysis.

\begin{tabular}{|c|c|c|}
\hline Primer name & Sequence & Purpose \\
\hline SpCas9 gRNA1 FWD & ACCGCAAGAAGTACAGCATCGGCC & sgRNA cloning \\
\hline SpCas9 gRNA1 REV & AACGGCCGATGCTGTACTTCTTGC & sgRNA cloning \\
\hline SpCas9 gRNA2 FWD & ACCGTACAGCATCGGCCTGGACAT & sgRNA cloning \\
\hline SpCas9 gRNA2 REV & AACATGTCCAGGCCGATGCTGTAC & sgRNA cloning \\
\hline SpCas9 gRNA3 FWD & ACCGCCGATGCTGTACTTCTTGT & sgRNA cloning \\
\hline SpCas9 gRNA3 REV & AACACAAGAAGTACAGCATCGGC & sgRNA cloning \\
\hline SpCas9 gRNA4 FWD & ACCGCAGAGTTGGTGCCGATGTCC & sgRNA cloning \\
\hline SpCas9 gRNA4 REV & AACGGACATCGGCACCAACTCTGC & sgRNA cloning \\
\hline U6p-Mlul FWD & AGCACGCGTGAGGGCCTATTTCCCATGAT & Vector construct \\
\hline $\begin{array}{l}\text { SpCas9 sgRNA } \\
\text { scaffold-Mlul REV }\end{array}$ & GCTACGCGTAAAAAAAGCACCGACTCGGT & Vector construct \\
\hline YFP sgRNA2 FWD & CACCGCGAGGAGCTGTTCACCGGGG & sgRNA cloning \\
\hline YFP sgRNA2 REV & AAACCCCGGTGAACAGCTCCTCGC & sgRNA cloning \\
\hline YPF sgRNA6 FWD & ACCGCGTCGCCGTCCAGCTCGACC & sgRNA cloning \\
\hline
\end{tabular}




\begin{tabular}{|lll|}
\hline YPF sgRNA6 REV & AACGGTCGAGCTGGACGGCGACGC & SgRNA cloning \\
\hline SpCas9 FWD & TACGCTTCGCCGAAGAAAAGC & qPCR \\
\hline SpCas9 REV & GTGTTGCCCAGCACCTTGAATT & qPCR \\
\hline mCherry FWD & CCGACATCCCCGACTACTTGAA & qPCR \\
\hline mCherry REV & TGTAGATGAACTCGCCGTCCTG & qPCR \\
\hline U6-Seq REV & GCGGCCGCACGCGTGAGGGC & Sequencing \\
\hline pX551-FWD & CCGAAGAGGTCGTGAAGAAG & qPCR \\
\hline pX551-REV & & qPCR \\
\hline pX552-FWD & TGTGGAAAGGACGAACACC & T7E1 PCR \\
\hline pX552-REV & TGGTCCTAAAACCCACTTGC & Sequencing \\
\hline CMV Seq-FWD & CGCAAATGGGCGGTAGGCGTG \\
\hline
\end{tabular}




\section{Supplementary Figure 1}

YFP SgRNA

Cas9 sgRNA/YFP sgRNA
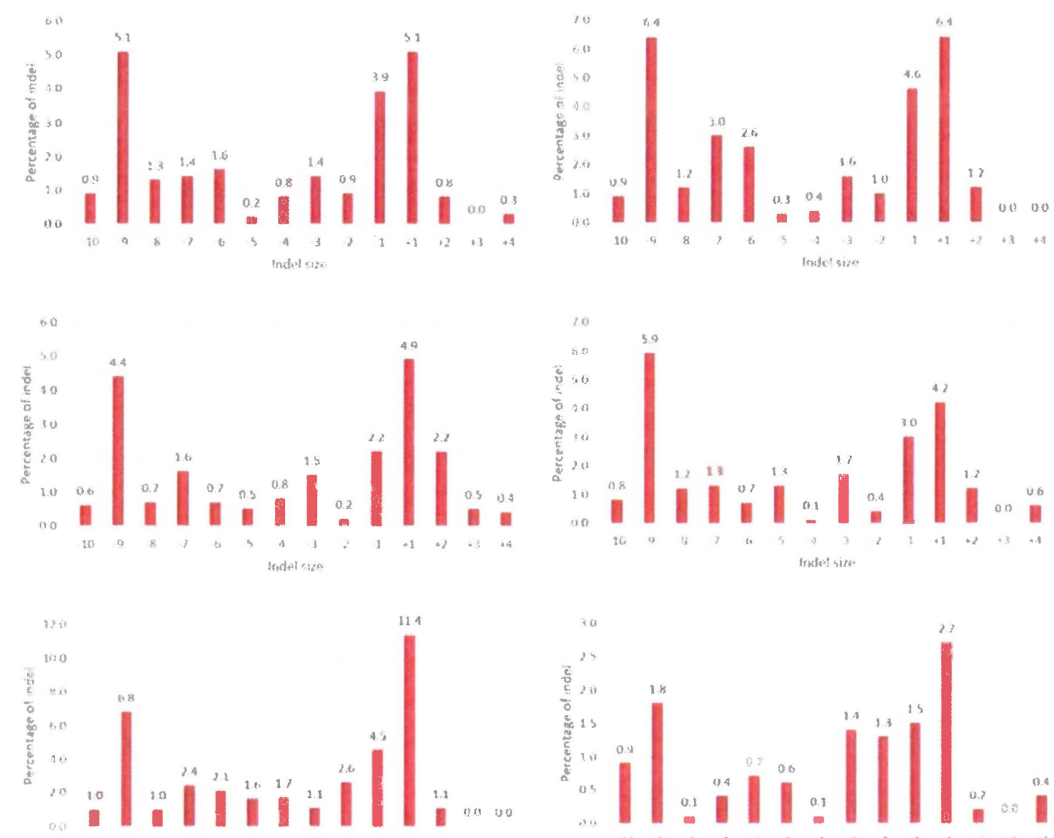

Supplementary Figure 1. Spectrum and frequency of indels induced by conventional (YFP sgRNA; $n=3$ ) or kamikaze CRISPR/Cas systems (Cas9 sgRNA/YFP-sgRNA; $n=3$ ) for knockout of YFP in HEK293-YFP cell line were determined using the inference of CRISPR edits (ICE) tool (https://ice.synthego.com/\#/). 


\section{Supplementary Figure 2}

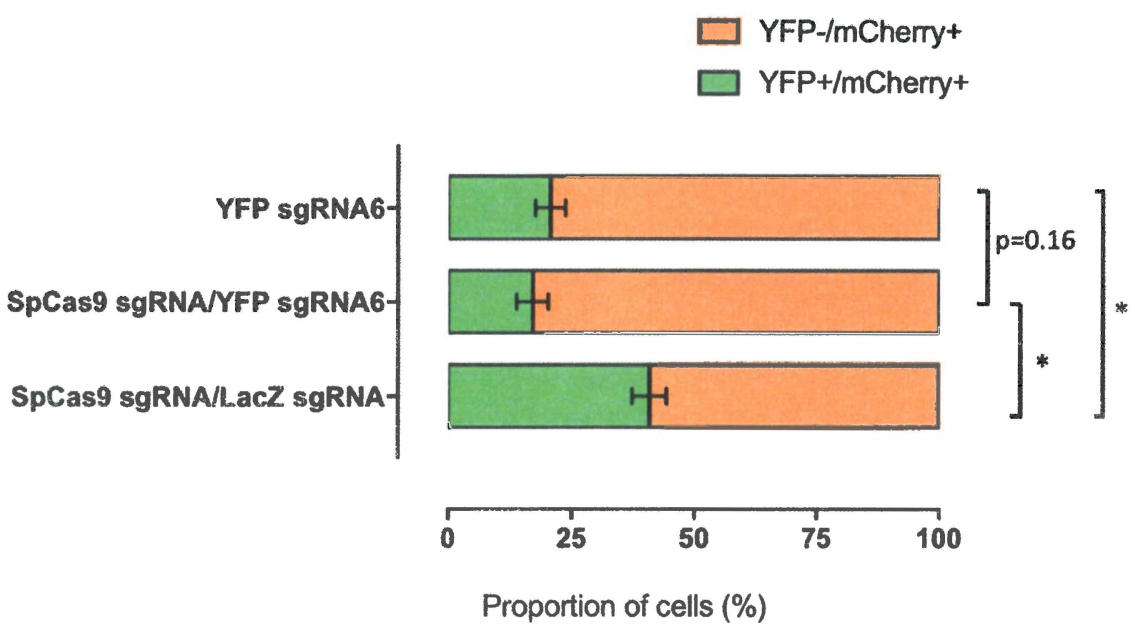

Supplementary Figure 2. Quantification of YFP disruption in the retina. The percentage of YFP disruption following AAV2-mediated delivery of SpCas9 sgRNA/YFP sgRNA6, YFP SgRNA6 or SpCas9 sgRNA/LacZ sgRNA was assessed by manual cell counting. Representative data are shown for 3-5 retinas and expressed as the Mean \pm SEM. Statistical analysis between groups was performed using one-way ANOVA followed by Tukey's multiple comparisons test $(* p<0.05)$. 
Page 31 of 41

Supplementary Figure 3

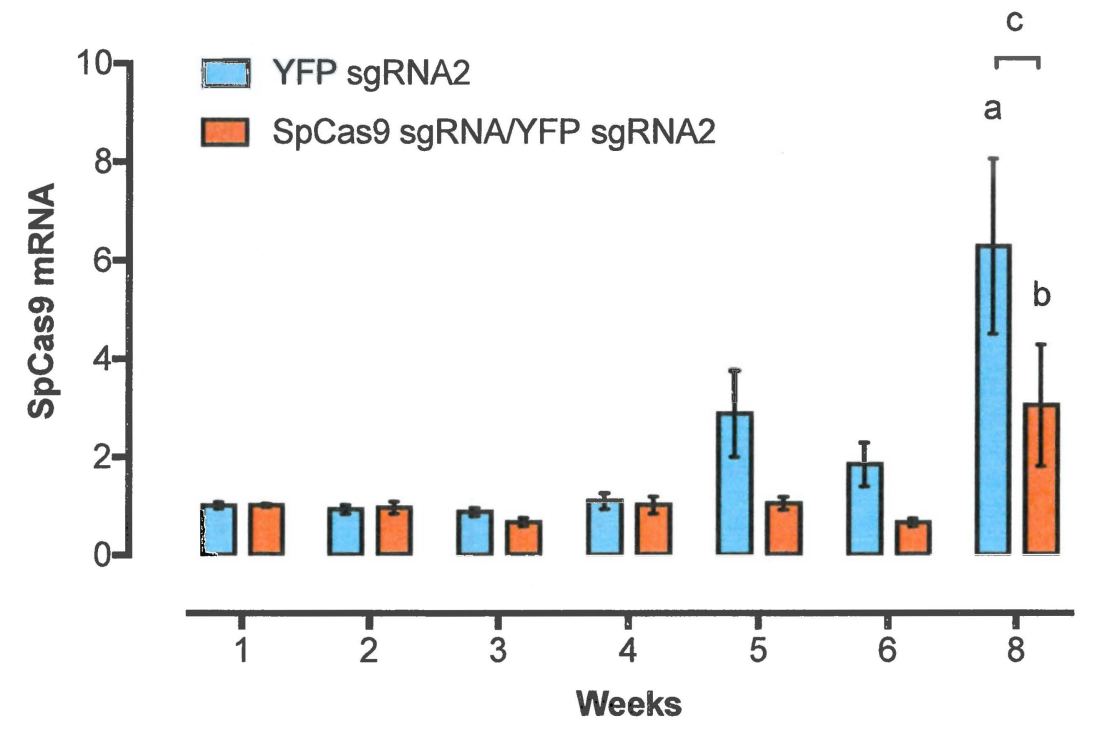

Supplementary Figure 3. Time course of SpCas9 mRNA expression in the mouse retina. SpCas9 mRNA were isolated from the mouse retina administrated with AAV2-SpCas9 sgRNA/YFP sgRNA2 or AAV2-YFP sgRNA2 at 1, 2, 3, 4, 5, 6 and 8 weeks after intravitreal injection. Relative fold change of SpCas9 expression was normalized by week 1 from each treatment group. Representative data are shown for 5-6 retinas per group/time point and expressed as Mean \pm SEM. Statistical analysis between groups was performed using Twoway ANOVA followed by Sidak's multiple comparisons test. $a$, YFP sgRNA2: 1 vs 8 weeks, $\mathrm{p}=0.002$. $b$, SpCas9/YFP sgRNA: 1 vs 8 weeks, $\mathrm{p}=0.7043$. $c$, YFP sgRNA2 vs SpCas9/YFP sgRNA, $p=0.0142$. 


\section{Supplementary Figure 4}

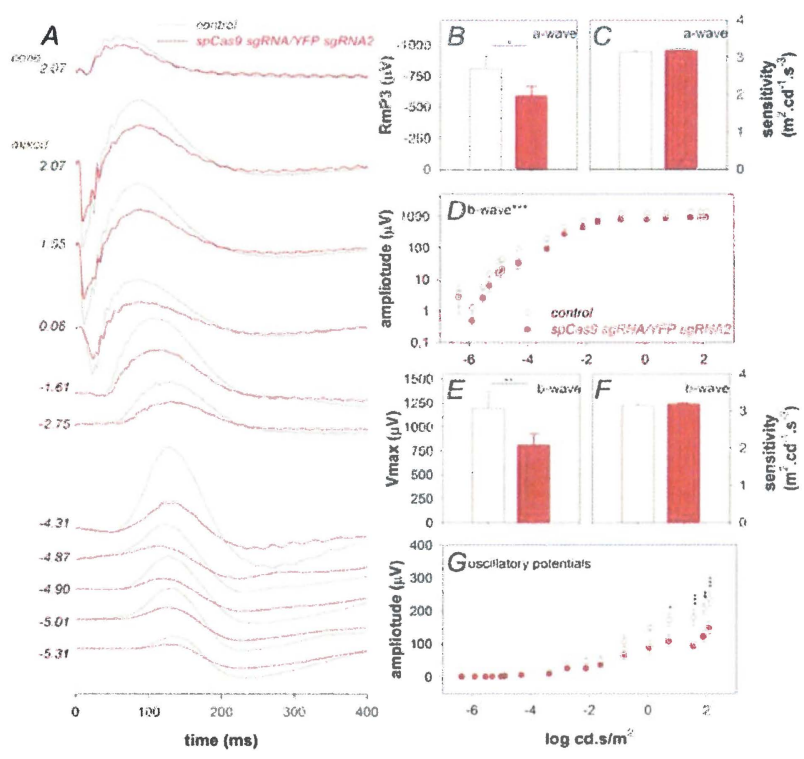

Supplementary Figure 4. SpCas9 sgRNA/YFP sgRNA2 decreased retinal function. (A) Averaged ERG waveforms at selected intensities for control ( $n=4$, black) and treated eyes $(n=4$, red). (B) Groups average photoreceptoral (a-wave) saturated amplitude for contralateral control (unfilled) and treated eyes (filled). (C) Photoreceptoral sensitivity to light. (D) Intensity response characteristics across the entire range of intensities. (E) Bipolar cell amplitude. (F) Bipolar cell sensitivity to light. (G) Inner retinal amacrine cell mediated response (oscillatory potentials). Data are expressed as the Mean \pm SEM. Statistical analysis between groups was performed using two-tailed Student's t-test. Asterisks denotes significance $* P<0.05, * * P<0.01, * * * P<0.001$. 


\section{Supplementary Figure 5}

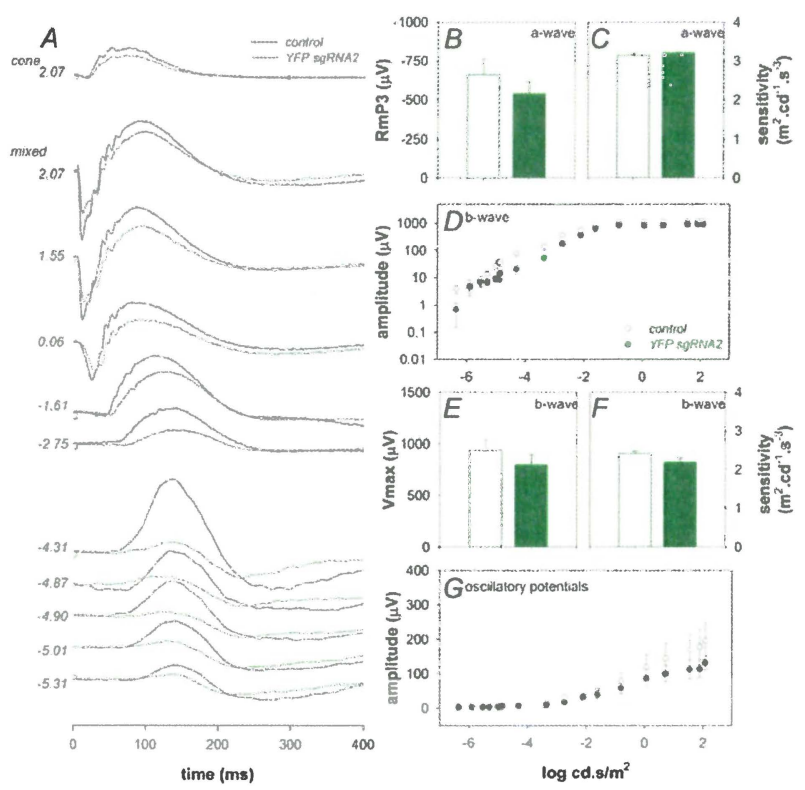

Supplementary Figure 5 YFP sgRNA2 alone affects inner retinal function. (A) Averaged ERG waveforms at selected intensities for control ( $n=5$, black) and treated eyes ( $n=5$, green). (B) Groups average ( \pm SEM) photoreceptoral (a-wave) saturated amplitude for contralateral control (unfilled) and treated eyes (filled). (C) Photoreceptoral sensitivity to light. (D) Intensity response characteristics across the entire range of intensities. (E) Bipolar cell amplitude. (F) Bipolar cell sensitivity to light. (G) Inner retinal amacrine cell mediated response (oscillatory potentials). Data are expressed as the Mean \pm SEM. Statistical analysis between groups was performed using two-tailed Student's t-test. 


\section{Supplementary Figure 6}

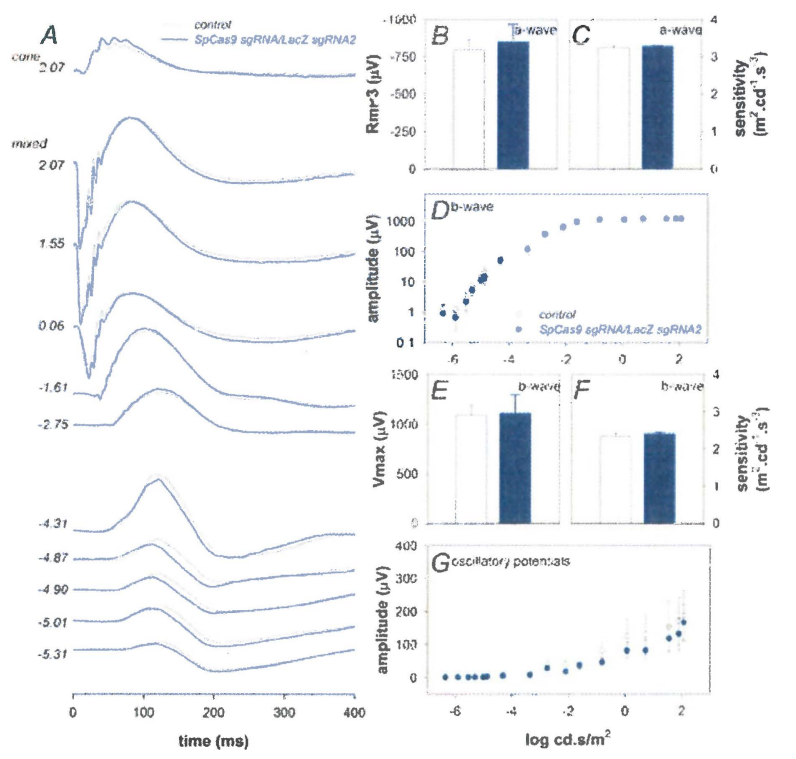

Supplementary Figure 6. SpCas9 sgRNA/LacZ sgRNA does not affect retinal function. (A) Averaged ERG waveforms at selected intensities for control ( $n=3$, black) and treated eyes $(n=3$, blue). (B) Groups average $( \pm S E M)$ photoreceptoral (a-wave) saturated amplitude for contralateral control (unfilled) and treated eyes (filled). (C) Photoreceptoral sensitivity to light. (D) Intensity response characteristics across the entire range of intensities. (E) Bipolar cell amplitude. (F) Bipolar cell sensitivity to light. (G) Inner retinal amacrine cell mediated response (oscillatory potentials). Data are expressed as the Mean \pm SEM. Statistical analysis between groups was performed using two-tailed Student's t-test. 


\section{Supplementary Figure 7}
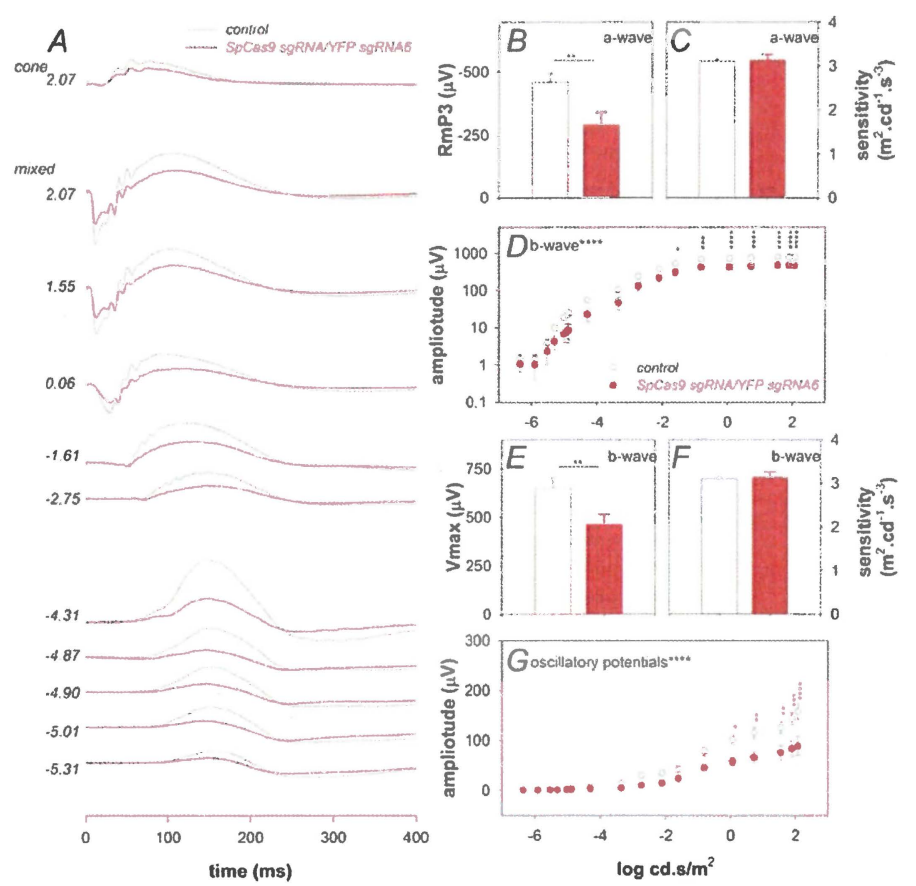

Supplementary Figure 7. SpCas9 sgRNA/YFP sgRNA6 decreased retinal function. (A) Averaged ERG waveforms at selected intensities for control ( $n=10$, black) and treated eyes $(n=10$, red). (B) Groups average ( $\pm S E M)$ photoreceptoral (a-wave) saturated amplitude for contralateral control (unfilled) and treated eyes (filled). (C) Photoreceptoral sensitivity to light. (D) Intensity response characteristics across the entire range of intensities. (E) Bipolar cell amplitude. (F) Bipolar cell sensitivity to light. (G) Inner retinal amacrine cell mediated response (oscillatory potentials). Data are expressed as the Mean \pm SEM. Statistical analysis between groups was performed using two-tailed Student's t-test. Asterisks denotes significance $* P<0.05, * * P<0.01, * * * P<0.001, * * * * P<0.0001$. 


\section{Supplementary Figure 8}

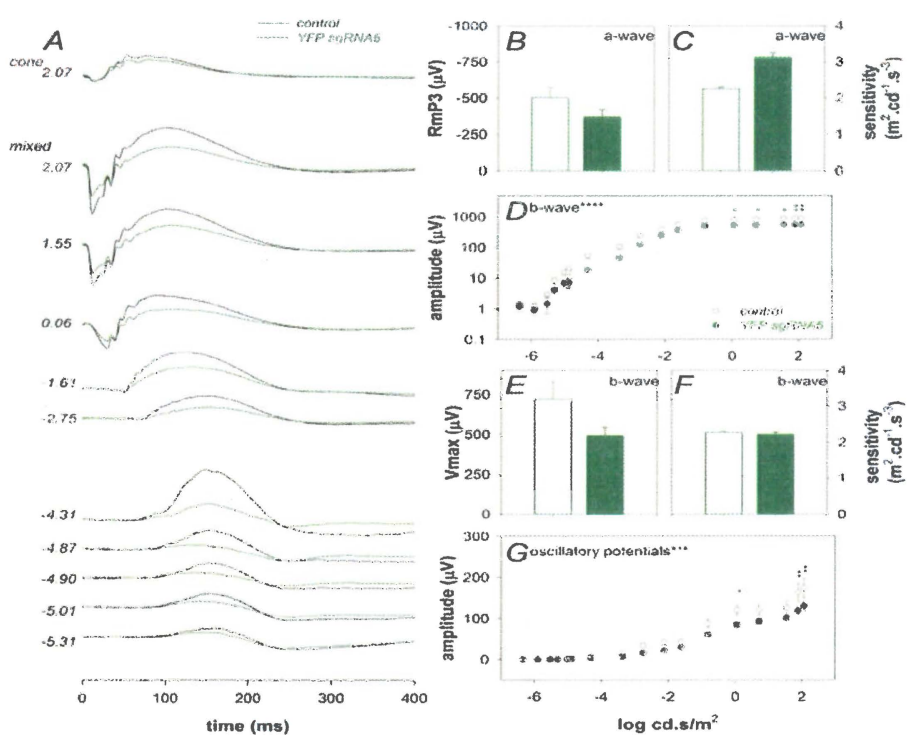

Supplementary Figure 8. YFP sgRNA6 alone affects inner retinal function. (A) Averaged ERG waveforms at selected intensities for control ( $n=8$, black) and treated eyes ( $n=8$, green). (B) Groups average photoreceptoral (a-wave) saturated amplitude for contralateral control (unfilled) and treated eyes (filled). (C) Photoreceptoral sensitivity to light. (D) Intensity response characteristics across the entire range of intensities. (E) Bipolar cell amplitude. (F) Bipolar cell sensitivity to light. (G) Inner retinal amacrine cell mediated response (oscillatory potentials). Data are expressed as the Mean \pm SEM. Statistical analysis between groups was performed using two-tailed Student's t-test. Asterisks denotes significance. ${ }^{*} \mathrm{P}<0.05, * * \mathrm{P}<0.01$. 
Supplementary Figure 9

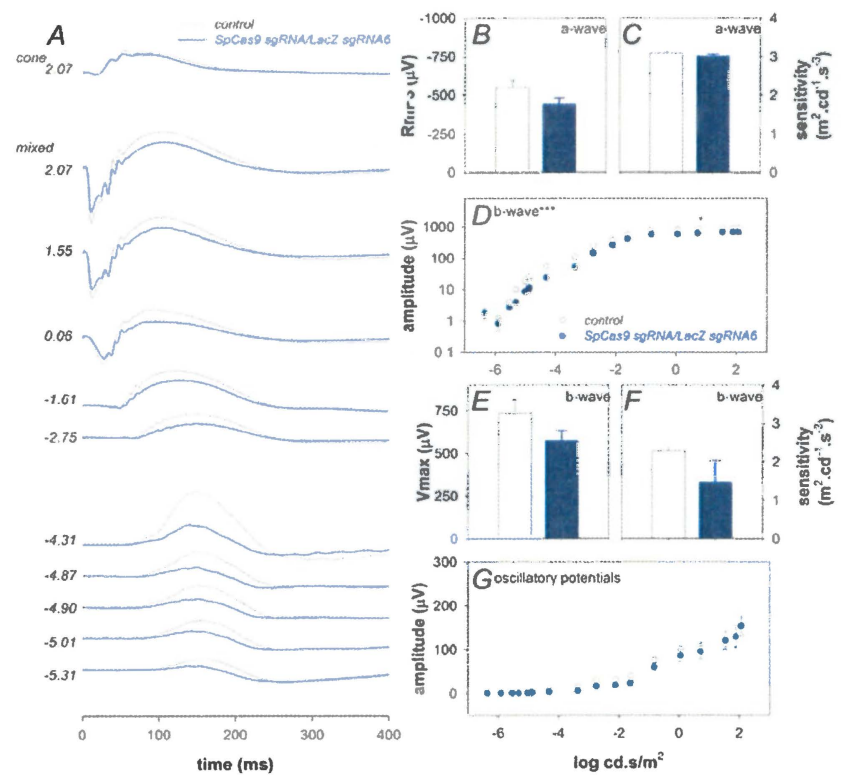

Supplementary Figure 9. SpCas9 sgRNA/LacZ sgRNA does not affect retinal function. (A) Averaged ERG waveforms at selected intensities for control ( $n=8$, black) and treated eyes ( $n=8$, blue). (B) Groups average photoreceptoral (a-wave) saturated amplitude for contralateral control (unfilled) and treated eyes (filled). (C) Photoreceptoral sensitivity to light. (D) Intensity response characteristics across the entire range of intensities. (E) Bipolar cell amplitude. (F) Bipolar cell sensitivity to light. (G) Inner retinal amacrine cell mediated response (oscillatory potentials). Data are expressed as the Mean \pm SEM. Statistical analysis between groups was performed using two-tailed Student's t-test. 

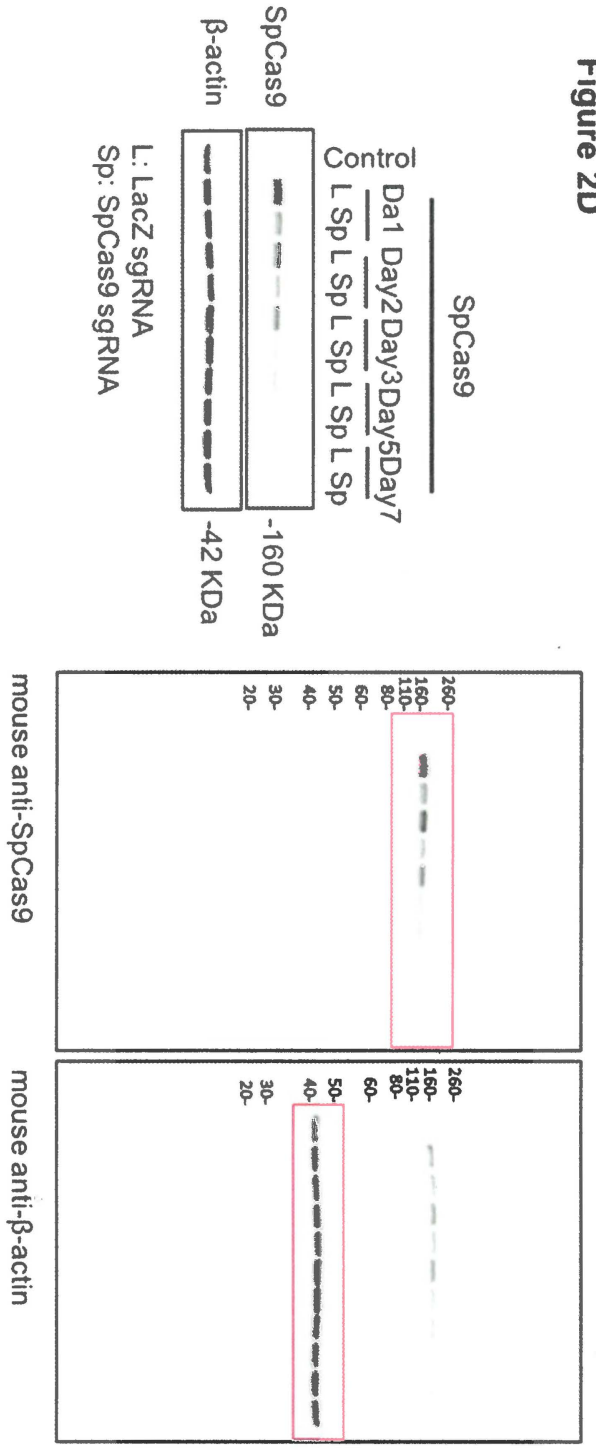

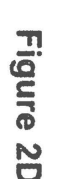

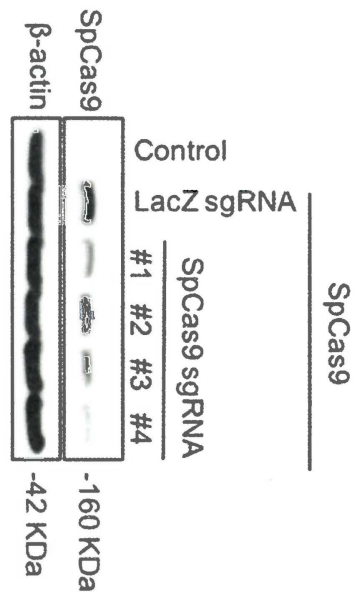

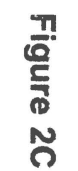

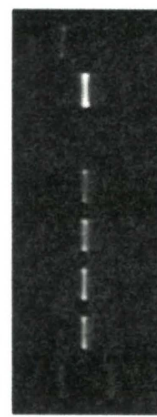

Uncut control

Cut control

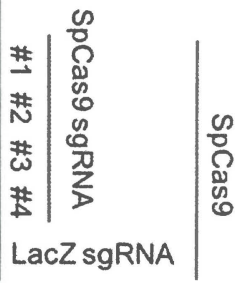

$\stackrel{\dot{1}}{\dot{\hat{\sigma}}}$

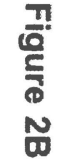
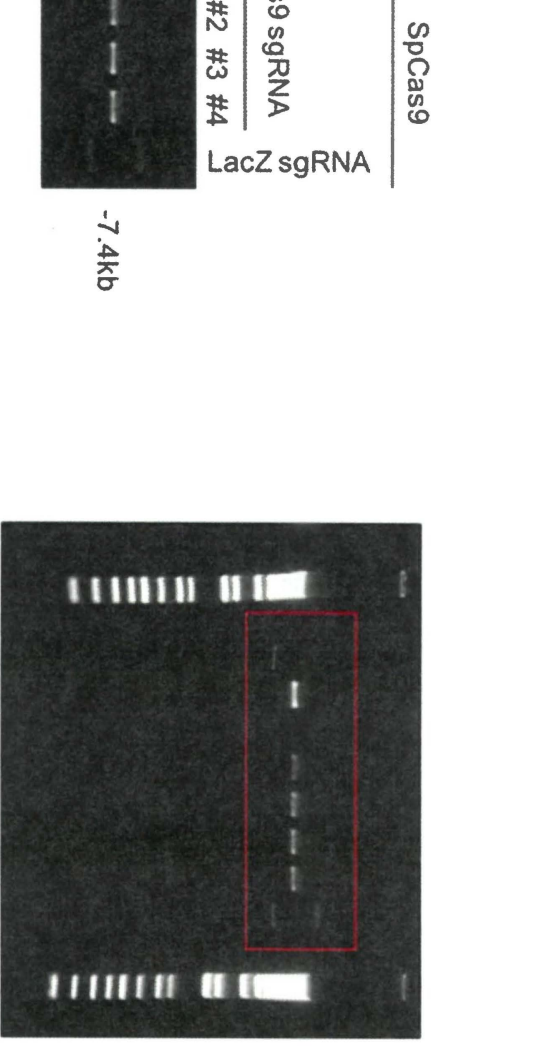

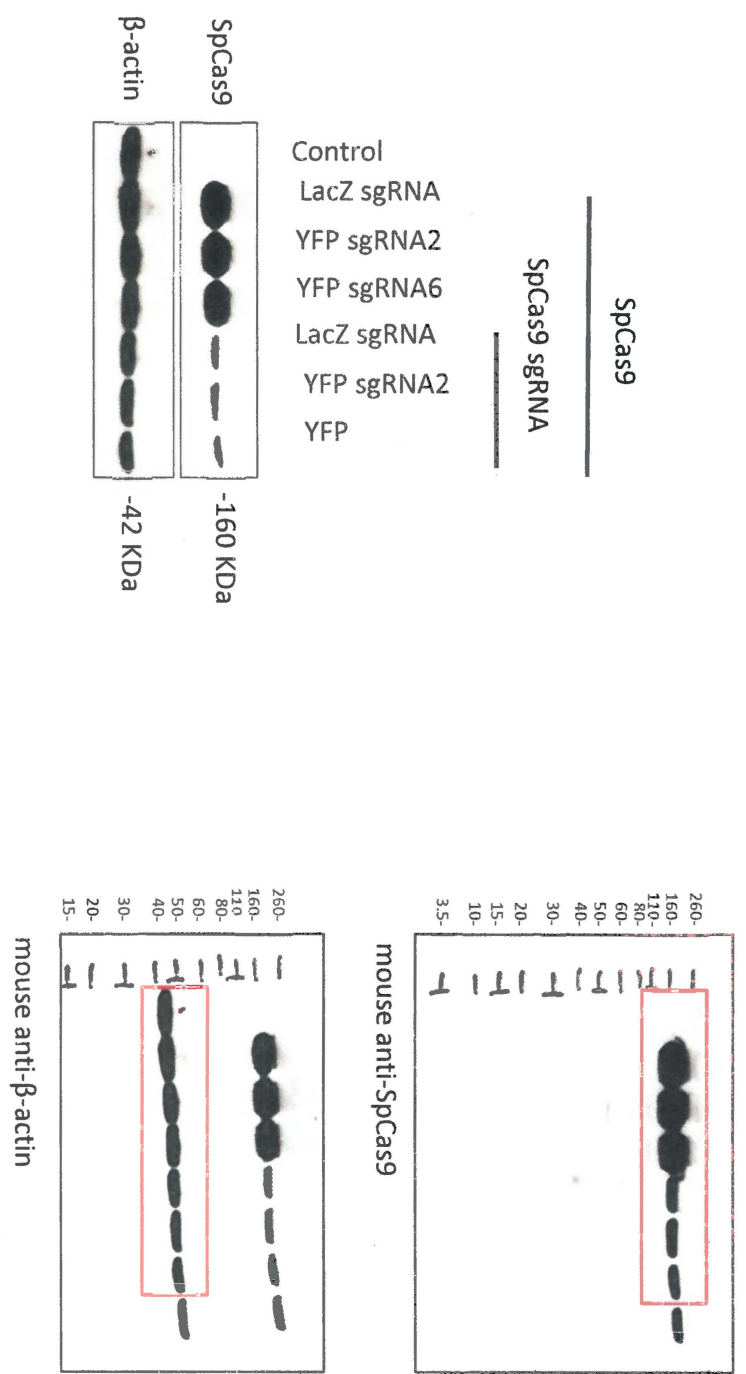

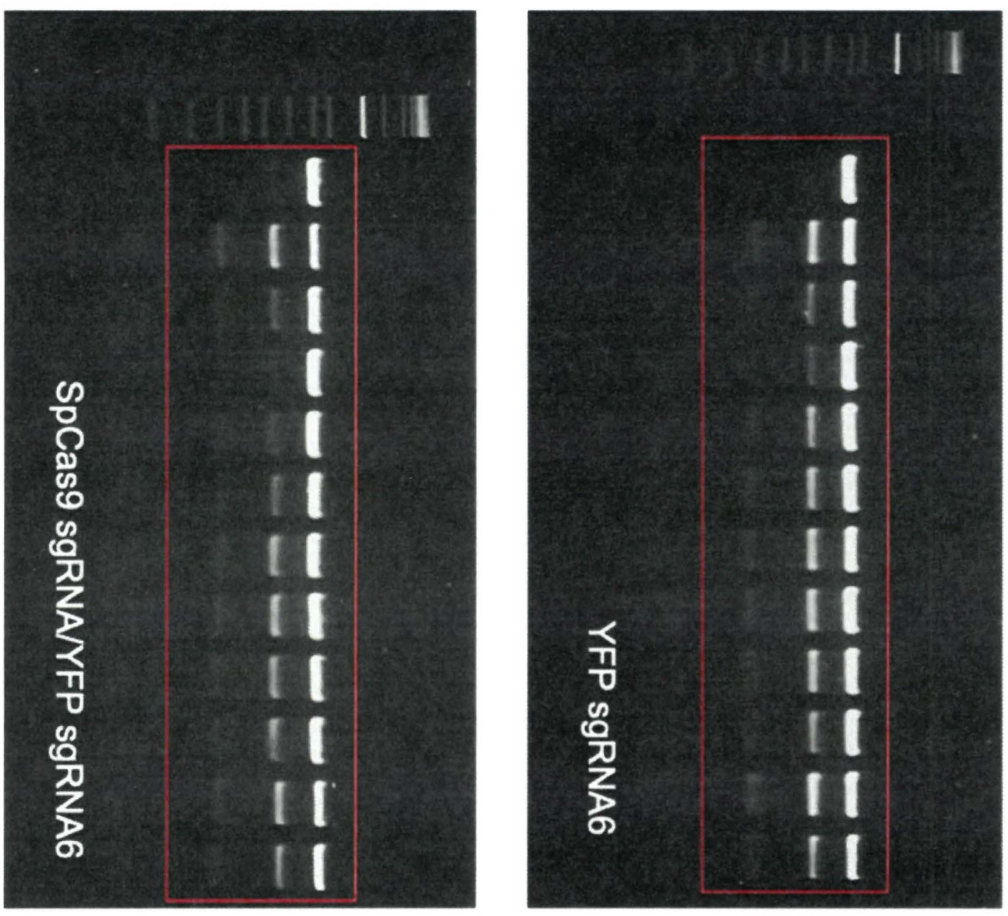

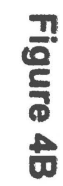
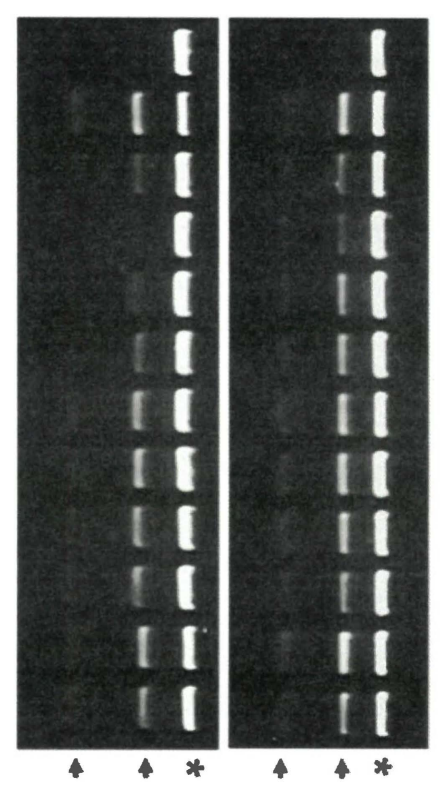

अवृ

20

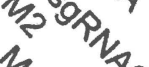

多

\%

$\xi_{0}$

$\xi$

గ్

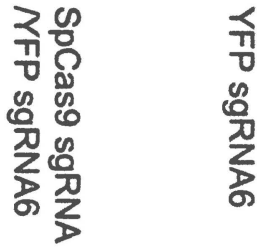

